\title{
WEIGHTED KORN AND POINCARÉ-KORN INEQUALITIES IN THE EUCLIDEAN SPACE AND ASSOCIATED OPERATORS
}

\author{
KLEBER CARRAPATOSO, JEAN DOLBEAULT, FRÉDÉRIC HÉRAU, STÉPHANE MISCHLER, \\ AND CLÉMENT MOUHOT
}

\begin{abstract}
We prove functional inequalities on vector fields $u: \mathbb{R}^{d} \rightarrow \mathbb{R}^{d}$ when $\mathbb{R}^{d}$ is equipped with a bounded measure $e^{-\phi} \mathrm{d} x$ that satisfies a Poincaré inequality, and study associated self-adjoint operators. The weighted Korn inequality compares the differential matrix $D u$, once projected orthogonally to certain finite-dimensional spaces, with its symmetric part $D^{s} u$ and, in an improved form of the inequality, an additional term $\nabla \phi \cdot u$. We also consider Poincaré-Korn inequalities for estimating a projection of $u$ by $D^{s} u$ and zeroth-order versions of these inequalities obtained using the Witten-Laplace operator. The constants depend on geometric properties of the potential $\phi$ and the estimates are quantitative and constructive. These inequalities are motivated by kinetic theory and related with the Korn inequality (1906) in mechanics, which compares $D u$ and $D^{s} u$ on a bounded domain.
\end{abstract}

\section{Introduction AND MAIN RESUlts}

1.1. The problem at hand. Korn's inequality $[28,29,30]$ is a classical tool in continuum mechanics which asserts the control of the $L^{2}$ norm of the gradient of a vector field defined on a smooth bounded domain $\Omega$ of $\mathbb{R}^{d}$ by the $L^{2}$ norm of its symmetric part:

$$
\|D u\|_{L^{2}(\Omega)}^{2} \leq 2\left\|D^{s} u\right\|_{L^{2}(\Omega)}^{2}, \quad \forall u \in C^{2}\left(\bar{\Omega} ; \mathbb{R}^{d}\right) \text { such that } u=0 \text { on } \partial \Omega .
$$

If $\mathfrak{M}$ (resp. $\mathfrak{M}^{s}$ ) is the set of $d \times d$ real (resp. symmetric) matrices, $D u$ (resp. $D^{s} u$ ) is the differential of $u$ (resp. its symmetric part) and takes values in $\mathfrak{M}$ (resp. $\left.\mathfrak{M}^{s}\right)$. Written with cartesian coordinates, this means

$$
\left(D^{s} u\right)_{i j}=\frac{1}{2}\left(\partial_{j} u_{i}+\partial_{i} u_{j}\right) \quad \text { and } \quad\left(D^{a} u\right)_{i j}=\frac{1}{2}\left(\partial_{j} u_{i}-\partial_{i} u_{j}\right)
$$

We denote by $A^{a} \in \mathfrak{M}^{a}$ the skew-symmetric part of $A \in \mathfrak{M}$ and by $A^{s}=A-A^{a}$ its symmetric part. The original proof of (1) in [28] is instructive: it is enough to integrate over $\Omega$ the pointwise identities

$$
\left|D^{a} u\right|^{2}-\left|D^{s} u\right|^{2}+(\nabla \cdot u)^{2}-\nabla \cdot[u(\nabla \cdot u)-(u \cdot \nabla) u]=0 \quad \text { and } \quad|D u|^{2}=\left|D^{s} u\right|^{2}+\left|D^{a} u\right|^{2}
$$

and use the boundary condition to get $2\left\|D^{s} u\right\|_{L^{2}(\Omega)}^{2}=\|D u\|_{L^{2}(\Omega)}^{2}+\|\nabla \cdot u\|_{L^{2}(\Omega)}^{2}$, where $\nabla \cdot u$ denotes the divergence of $u$ and $L^{2}(\Omega)$ is the $L^{2}$ norm for matrix-valued, vector-valued or real-valued functions.

The boundary condition $u=0$ is a severe restriction. In view of applications in kinetic theory, Desvillettes and Villani in [12] enlarge the set of possible vector fields to those satisfying only $u \cdot \mathbf{n}=0$ on the boundary, where $\mathbf{n}$ denotes the outward normal unit vector to $\partial \Omega$. The set of infinitesimal rotations

$$
\mathcal{R}:=\left\{R: x \in \mathbb{R}^{d} \mapsto A x \in \mathbb{R}^{d} \text { with } A \in \mathfrak{M}^{a}\right\}
$$

is a family of vector fields which plays a key role. If $\Omega$ has some rotational invariance, then some infinitesimal rotations satisfy the boundary condition while their symmetric differential is zero. Being invariant under the action of a group of rotations $t \rightarrow e^{t A}$ for a given $A \in \mathfrak{M}^{a}$ means that

$$
\forall t \in \mathbb{R}, \quad e^{t A} \Omega=\Omega
$$

(here we suppose that $\Omega$ is invariant under rotations centred at 0 without loss of generality). Taking the derivative with respect to $t$ shows that the set of infinitesimal rotations preserving $\Omega$ is

$$
\mathcal{R}_{\Omega}:=\{R \in \mathcal{R}: \forall x \in \partial \Omega, \mathbf{n}(x) \cdot R(x)=0\},
$$

Date: December 14, 2020

2020 Mathematics Subject Classification. Primary: 49J40. Secondary: 46E35, 49Q20.

Key words and phrases. Korn inequality; weighted Poincaré inequality; Poincaré-Korn inequality; Lions' lemma; WittenLaplace operator; Grad's number. 
where we implicitly use the fact that skew-symmetric matrices generate the tangent space of the orthogonal group. In [12, Inequality (38)], Desvillettes and Villani state the following Korn inequality

$$
\inf _{R \in \mathcal{R}_{\Omega}}\|D(u-R)\|_{L^{2}(\Omega)}^{2} \leq C_{\Omega}\left\|D^{s} u\right\|_{L^{2}(\Omega)}^{2}, \quad \forall u \in C^{2}\left(\bar{\Omega} ; \mathbb{R}^{d}\right) \text { such that } u \cdot \mathbf{n}=0 \text { on } \partial \Omega,
$$

which takes into account invariances by rotation. They obtain quantitative estimates on the constant $C_{\Omega}$. Inequality (3) is an important ingredient in [13] to prove hypocoercivity for the Boltzmann equation in $\Omega$.

In this article, our aim is to establish similar Korn and related inequalities, with constructive constants, in the whole Euclidean space $\mathbb{R}^{d}$ in presence of a confining potential $\phi: \mathbb{R}^{d} \rightarrow \mathbb{R}$, i.e., in the $L^{2}$ space with reference measure $e^{-\phi(x)} \mathrm{d} x$. Our motivation comes from the hypocoercivity theory of kinetic operators with more than one microscopic invariant studied in [7], but the inequalities are of independent interest.

1.2. Assumptions and notations. We consider a potential $\phi: \mathbb{R}^{d} \rightarrow \mathbb{R}, d \geq 2$ satisfying the conditions:

(1) the measure $e^{-\phi(x)} \mathrm{d} x$ is a centred probability measure

$$
\int_{\mathbb{R}^{d}} e^{-\phi(x)} \mathrm{d} x=1 \text { and } \int_{\mathbb{R}^{d}} x e^{-\phi(x)} \mathrm{d} x=0,
$$

(2) the potential $\phi$ is of class $C^{2}\left(\mathbb{R}^{d} ; \mathbb{R}\right)$ and, for all $\varepsilon>0$, there exist a constant $C_{\varepsilon}$ such that

$$
\forall x \in \mathbb{R}^{d}, \quad\left|D^{2} \phi(x)\right| \leq \varepsilon|\nabla \phi(x)|^{2}+C_{\varepsilon},
$$

(3) the measure $e^{-\phi} \mathrm{d} x$ satisfies the Poincaré inequality with constant $C_{P}$ : for all scalar functions $f$ in the space $\mathcal{C}_{c}^{\infty}\left(\mathbb{R}^{d} ; \mathbb{R}\right)$ of smooth functions with compact support, we have

$$
\int_{\mathbb{R}^{d}}|f(x)-\langle f\rangle|^{2} e^{-\phi(x)} \mathrm{d} x \leq C_{\mathrm{P}} \int_{\mathbb{R}^{d}}|\nabla f(x)|^{2} e^{-\phi(x)} \mathrm{d} x, \quad \text { where } \quad\langle f\rangle:=\int_{\mathbb{R}^{d}} f(x) e^{-\phi(x)} \mathrm{d} x .
$$

Assumption (H1) is a classical integrability condition on $\phi$. The fact that the center of mass $\int_{\mathbb{R}^{d}} x e^{-\phi(x)} \mathrm{d} x$ is finite is a consequence of (H3) applied with $f(x)=x_{i}, i=1, \ldots, d$. There is no loss of generality in choosing $\int_{\mathbb{R}^{d}} x e^{-\phi(x)} \mathrm{d} x=0$. Assumption (H2) is a regularity assumption at infinity which, in the language of operator theory and in a suitable functional framework, says that the multiplication operator by $\left|D^{2} \phi(x)\right|$ is infinitesimally bounded by the multiplication operator by $|\nabla \phi(x)|^{2}$ (see [37, Chapter X]). Here no growth assumption is made on $|\nabla \phi|$. Assumption (H2) is satisfied for instance if

$$
\sup _{x \in \mathbb{R}^{d}} \frac{D^{2} \phi(x)}{\sqrt{1+|\nabla \phi(x)|^{2}}}<\infty \quad \text { or } \quad \lim _{|x| \rightarrow \infty} \frac{D^{2} \phi(x)}{1+|\nabla \phi(x)|^{2}}=0 .
$$

Assumption (H3) can be interpreted as a measure concentration property: it implies that

$$
\int_{\mathbb{R}^{d}}|\nabla \phi(x)|^{2} e^{-\phi(x)} \mathrm{d} x<\infty \quad \text { and } \quad \forall k \in \mathbb{N}, \quad \int_{\mathbb{R}^{d}}|x|^{2 k} e^{-\phi(x)} \mathrm{d} x<\infty
$$

by (H2) for the first estimate and by an easy induction (see Remark 11 in Appendix A.1) for the second one. Assumptions (H1), (H2) and (H3) are satisfied, for instance, if $\phi \in C^{2}\left(\mathbb{R}^{d} ; \mathbb{R}\right)$ and either $\phi(x)=\alpha|x|^{\gamma}+\beta$ with $\gamma \geq 1$ or $\phi(x)=\alpha e^{|x|^{2}}+\beta$, for large values of $|x|$, where $\alpha>0$ and $\beta$ are two parameters. The three assumptions are also satisfied by the normalized Gaussian defined by

$$
\forall x \in \mathbb{R}^{d}, \quad \phi(x)=\frac{1}{2}|x|^{2}+\frac{d}{2} \ln (2 \pi) .
$$

Associated with $\phi$ and thanks to (H2), there exists two constants $C_{\phi}>8$ and $C_{\phi}^{\prime}>C_{\phi}$ such that

$$
\forall x \in \mathbb{R}^{d}, \quad 4 \sqrt{d}\left|D^{2} \phi(x)\right| \leq|\nabla \phi(x)|^{2}+C_{\phi}-1 \quad \text { and } \quad 4\left|D^{2} \phi(x)\right| \leq C_{\phi}^{-1 / 2}\left(|\nabla \phi(x)|^{2}+C_{\phi}^{\prime}\right) .
$$

As in the case of a bounded domain, the set $\mathcal{R}$ of infinitesimal rotations plays a key role in the study of Korn inequalities in the whole space. The symmetric differential applied to an infinitesimal rotation is zero. In our setting, the invariance under the action of a group of rotations $t \rightarrow e^{t A}$ for a given $A \in \mathfrak{M}^{a}$ means

$$
\forall t \in \mathbb{R}, \quad \forall x \in \mathbb{R}^{d}, \quad \phi\left(e^{t A} x\right)=\phi(x),
$$

where, again, we implicitly use the assumption that the measure is centred. Differentiating the above identity with respect to $t$ yields that the set of infinitesimal rotations preserving $\phi$ is

$$
\mathcal{R}_{\phi}:=\left\{R \in \mathcal{R}: \forall x \in \mathbb{R}^{d}, \nabla \phi(x) \cdot R(x)=0\right\} .
$$


This set is a central geometric objet in our analysis. In the inequalities, the addition of a term involving $\nabla \phi \cdot R$ allows us to control the infinitesimal rotations for which $\phi$ is not invariant, as we shall se later.

In this article, we adopt the following conventions. We denote by $|\cdot|$ the Euclidean norm in $\mathbb{R}, \mathbb{R}^{d}$ and $\mathfrak{M}$, by $a \cdot b$ the scalar product of two vectors in $\mathbb{R}^{d}$ and by $A: B$ the scalar product of two matrices $A$ and $B$ seen as vectors in $\mathbb{R}^{d^{2}}$. We denote by $\|\cdot\|$ the $L^{2}$ norm corresponding to $|\cdot|$ and weight $e^{-\phi} \mathrm{d} x$, and by $(\cdot, \cdot)$ the corresponding scalar product, that is,

$$
(f, f)=\|f\|^{2}=\int_{\mathbb{R}^{d}}|f(x)|^{2} e^{-\phi} \mathrm{d} x
$$

and we will refer to $L^{2}$ indifferently for functions with values in $\mathbb{R}, \mathbb{R}^{d}$ and $\mathfrak{M}$. We shall use $\langle\cdot\rangle$ for the average (component by component) according to the measure $e^{-\phi} \mathrm{d} x$ of functions with values in $\mathbb{R}, \mathbb{R}^{d}$ and $\mathfrak{M}$. We use the notation $\nabla$ for the gradient of scalar functions (with values in $\mathbb{R}^{d}$ ) and $D$ for the gradient of vector fields (with values in $\mathfrak{M}$ ). We denote by $H^{1}$ the space of functions $f$ or (when there is no ambiguity) vector fields $u$ such that respectively $f$ and $\nabla f$ or $u$ and $D u$ are in $L^{2}$. The space $H^{-1}$ is the dual of $H^{1}$ with respect to the $L^{2}$ scalar product. The weight function $\lfloor\nabla \phi\rceil$ is defined by

$$
\lfloor\nabla \phi\rceil:=\sqrt{1+|\nabla \phi|^{2}} .
$$

Let $\mathbb{P}$ be the orthogonal projection of vector-valued functions in $L^{2}$ onto $\mathcal{R}$, and $\mathbb{P}_{\phi}$ the orthogonal projection onto $\mathcal{R}_{\phi}$. We denote by $\mathcal{R}_{\phi}^{\perp}$ the orthogonal vector space to $\mathcal{R}_{\phi}$ in $L^{2}$ and $\mathcal{R}_{\phi}^{c}=\mathcal{R} \cap \mathcal{R}_{\phi}^{\perp}$ the restriction of the orthogonal space to $\mathcal{R}_{\phi}$ in $\mathcal{R}$ or, in other words, $\mathcal{R}_{\phi}^{c}=\mathbb{P} \mathcal{R}_{\phi}^{\perp}$. For instance if $\phi$ has no invariance by any rotation $e^{t A}$ then $\mathcal{R}_{\phi}=\{0\}$, and if $\phi$ is radially symmetric then $\mathcal{R}_{\phi}=\mathcal{R}$. Let $\mathfrak{P}$ be the orthogonal projection of matrix-valued functions in $L^{2}$ onto the set of constant antisymmetric matrices $\mathfrak{M}^{a}=D \mathcal{R}$. For all vector field $u \in H^{1}$, we have

$$
\mathfrak{P}(D u)=\left\langle D^{a} u\right\rangle .
$$

We also denote by $\mathfrak{P}_{\phi}$ the $L^{2}$ orthogonal projector onto $\mathfrak{M}_{\phi}:=D \mathcal{R}_{\phi}$ and by $\mathfrak{M}_{\phi}^{c}$ the orthogonal of $\mathfrak{M}_{\phi}$ in $\mathfrak{M}^{a}$, i.e., $\mathfrak{M}_{\phi}^{c}=\mathfrak{P M}_{\phi}^{\perp}$. The projections are summarised in Figure 1.2. Note that $D \mathcal{R}_{\phi}^{c}$ and $\mathfrak{M}_{\phi}^{c}$ generically differ since the inner products underlying the two orthogonal decomposition are different.

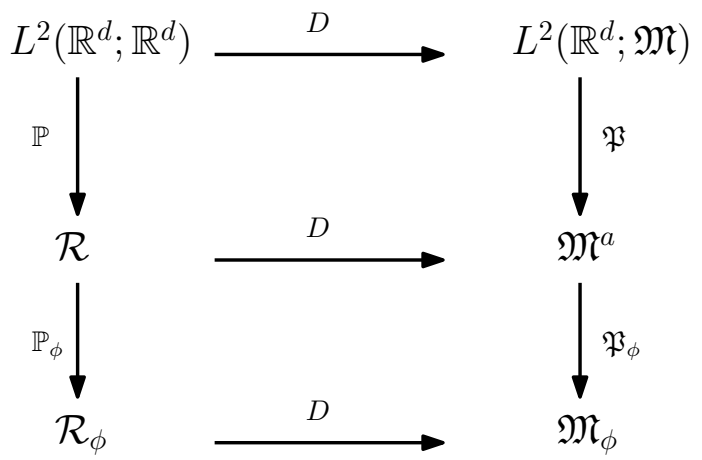

Figure 1. Representation of the orthogonal decompositions.

One additional notation will be used throughout this paper: if $x$ and $y$ are two vectors in $\mathbb{R}^{d}$, we denote by $x \otimes y$ the matrix $\left(x_{i} y_{j}\right)_{1 \leq i, j \leq d}$. Further details on $D \mathcal{R}_{\phi}^{c}$ and $\mathfrak{M}_{\phi}^{c}$ are collected in Appendix B.1.

1.3. Main results. All inequalities in this paper are quantitative with explicit estimates on the constants. The first result is the counterpart of (3) in the whole Euclidean space, with some additional consequences based on Poincaré inequalities. The statement involves the whole set of infinitesimal rotations $\mathcal{R}$.

Theorem 1 (Korn, Poincaré-Korn and strong Poincaré-Korn inequalities). Suppose (H1)-(H2)-(H3). Then there are a Korn constant $C_{\mathrm{K}}$, a Poincaré-Korn constant $C_{\mathrm{PK}}$ and a strong Poincaré-Korn constant $C_{\mathrm{SPK}}$ 
with explicit bounds involving only $C_{\mathrm{P}}, C_{\phi}$ and $C_{\phi}^{\prime}$ such that, for all $u \in H^{1}$,

$$
\begin{aligned}
& \inf _{R \in \mathcal{R}}\|D(u-R)\|^{2}=\|D u-\mathfrak{P}(D u)\|^{2} \leq C_{\mathrm{K}}\left\|D^{s} u\right\|^{2}, \\
& \inf _{R \in \mathcal{R}}\|u-\langle u\rangle-R\|^{2}=\|u-\langle u\rangle-\mathbb{P}(u)\|^{2} \leq C_{\mathrm{PK}}\left\|D^{s} u\right\|^{2}, \\
& \|\lfloor\nabla \phi\rceil(u-\langle u\rangle-\mathbb{P}(u))\|^{2} \leq C_{\mathrm{SPK}}\left\|D^{s} u\right\|^{2} .
\end{aligned}
$$

Moreover in the Gaussian case (5), optimal constants are $C_{\mathrm{K}}=4$, and $C_{\mathrm{PK}}=2$.

The terminology "Korn constant" refers to Korn's original results [28, 29, 30] whereas "Poincaré-Korn" and "strong Poincaré-Korn" respectively refer to usual Poincaré inequalities and to strong Poincaré inequalities (see Proposition 5). For brevity, we shall speak generically of "Korn-type inequalities" or simply "Korn inequalities". Explicit bounds for $C_{\mathrm{K}}, C_{\mathrm{PK}}$ and $C_{\mathrm{SPK}}$ will also be given later. The constant $C_{\mathrm{P}}$ is the optimal constant in (H3) while $C_{\phi}$ and $C_{\phi}^{\prime}$ refer to (6). The minimum in the left-hand side of (8) is explicit: according to (7), we have

$$
\|D u-\mathfrak{P}(D u)\|^{2}=\left\|D u-\left\langle D^{a} u\right\rangle\right\|^{2} \leq C_{\mathrm{K}}\left\|D^{s} u\right\|^{2} .
$$

In (9) and (10), there is no simple expression for $\mathbb{P}(u)$ as for $\mathfrak{P}(D u)$. Note that the strong Poincaré inequality (see Proposition 5 below) implies $\mathcal{R} \subset H^{1}$, so that the statement of Theorem 1 makes sense.

The defaults of axisymmetry of the boundary of the domain were taken into account in [12], in the bounded domain case without potential (this case will be called the "flat case" from now on). Here the eventual non-axisymmetry arises from the potential. Measuring the default of axisymmetry motivates our introduction of the finite dimensional space $\mathcal{R}_{\phi}^{c}$ and of the rigidity of vector fields constant $C_{\mathrm{RV}}$ defined by

$$
C_{\mathrm{RV}}^{-1}:=\min _{A x+b \in\left(\mathcal{R}_{\phi}^{c} \oplus \mathbb{R}^{d}\right) \backslash\{0\}} \frac{\|\nabla \phi(x) \cdot(A x+b)\|^{2}}{\|A x+b\|^{2}}
$$

if $\mathcal{R}_{\phi}^{c} \neq\{0\}$, and of the rigidity of differential constant $C_{\mathrm{RD}}$ defined by

$$
C_{\mathrm{RD}}^{-1}=\min _{(A, b) \in\left(\mathfrak{M}_{\phi}^{c} \otimes \mathbb{R}^{d}\right) \backslash\{(0,0)\}} \frac{\|\nabla \phi(x) \cdot(A x+b)\|^{2}}{|A|^{2}+|b|^{2}}
$$

if $\mathfrak{M}_{\phi}^{c} \neq\{0\}$. We adopt the convention that $C_{\mathrm{RV}}=0$ (respectively $C_{\mathrm{RD}}=0$ ) if $\mathcal{R}_{\phi}^{c}=\{0\}$ (respectively $\left.\mathfrak{M}_{\phi}^{c}=\{0\}\right)$. Let us show that these constants are well-defined in $\mathbb{R}_{+}$. Since $\mathcal{R}$ and $\mathfrak{M}_{\phi}$ have a finite dimension the minima in (11) and (12) exist. The first (respectively second) minimum is positive when $\mathcal{R}_{\phi}^{c} \neq\{0\}$ (respectively $\mathfrak{M}_{\phi}^{c} \neq\{0\}$ ). Indeed the linear maps $\mathcal{R}_{\phi}^{c} \oplus \mathbb{R}^{d}: A x+b \mapsto \nabla \phi(x) \cdot(A x+b) \in L^{2}$ and $\mathfrak{M}_{\phi}^{c} \oplus \mathbb{R}^{d}:(A, b) \mapsto \nabla \phi(x) \cdot(A x+b) \in L^{2}$ are injective: if $\nabla \phi(x) \cdot(A x+b)=0$ for all $x \in \mathbb{R}^{d}$, then by integration by parts

$$
0=\int_{\mathbb{R}^{d}} \nabla \phi(x) \cdot(A x+b) b \cdot x e^{-\phi(x)} \mathrm{d} x=|b|^{2}
$$

because $\langle x\rangle=0$, so that $b=0$, and as a consequence $A=0$ since $\mathcal{R}_{\phi} \cap \mathcal{R}_{\phi}^{c}=\{0\}$ and $\mathfrak{M}_{\phi} \cap \mathfrak{M}_{\phi}^{c}=\{0\}$. We can now state precised Korn and Poincaré-Korn inequalities in which $u$ is also controlled on the space of infinitesimal rotation that do not leave $\phi$ invariant.

Theorem 2 (Precised Poincaré-Korn and Korn inequalities). Suppose (H1)-(H2)-(H3). Then there are a precised Korn constant $C_{\mathrm{K}}^{\prime}$ and a precised Poincaré-Korn constant $C_{\mathrm{PK}}^{\prime}$ such that, for all $u \in H^{1}$,

$$
\begin{aligned}
& \inf _{R \in \mathcal{R}_{\phi}}\|u-R\|^{2}=\left\|u-\mathbb{P}_{\phi}(u)\right\|^{2} \leq C_{\mathrm{PK}}^{\prime}\left\|D^{s} u\right\|^{2}+2 C_{\mathrm{RV}}\|\nabla \phi \cdot u\|^{2}, \\
& \inf _{R \in \mathcal{R}_{\phi}}\|D(u-R)\|^{2}=\left\|D u-\mathfrak{P}_{\phi}(D u)\right\|^{2} \leq C_{\mathrm{K}}^{\prime}\left\|D^{s} u\right\|^{2}+2 C_{\mathrm{RD}}\|\nabla \phi \cdot u\|^{2} .
\end{aligned}
$$

Moreover the constants $C_{\mathrm{PK}}^{\prime}$ and $C_{\mathrm{K}}^{\prime}$ have explicit bounds depending only on the structural constants $C_{\mathrm{P}}$, $C_{\phi}, C_{\phi}^{\prime}, C_{\mathrm{RD}}$ and $C_{\mathrm{RV}}$.

Explicit bounds for $C_{\mathrm{K}}^{\prime}$ and $C_{\mathrm{PK}}^{\prime}$ will be given in the proofs. For any $u \in H^{1}$, the strong Poincaré inequality implies $\nabla \phi \cdot u \in L^{2}$ thanks to (4), so that the statement makes sense (see Proposition 5 below and Remarks 11 and 12 in Appendix A.1). The main difference with the flat case is the $\|\nabla \phi \cdot u\|^{2}$ term in the right-hand side of the inequality whereas there is no additional term in (3). This cannot be avoided as shown by the following example. Let us consider an infinitesimal rotation $u=R$, with $R \neq 0$, in the case without invariance by any rotation $e^{t A}$, that is, $\mathcal{R}_{\phi}=\{0\}$. Then $u \in H^{1}$ and inequality (14) reduces to 
$0 \neq\|R\|^{2} \leq 2 C_{\mathrm{RV}}\|\nabla \phi \cdot R\|^{2}$ since $D^{s} R=0$. This also shows that, compared to (8), an additional term is needed.

As often, the functional inequalities of Theorems 1 and 2 are linked with spectral properties of nonnegative differential operators. By a simple integration by parts, the formal adjoint of $\nabla$ in $L^{2}$ equipped with the weight $e^{-\phi(x)} \mathrm{d} x$ is $\nabla^{*} u=-\nabla_{\phi} \cdot u$ for any smooth vector field $u$, where $\nabla_{\phi} \cdot u:=\nabla \cdot u-\nabla \phi \cdot u$. The first operator is the so-called Witten-Laplace operator on functions $-\Delta_{\phi}$ (sometimes also called the OrnsteinUhlenbeck operator) which replaces the usual Laplacian in the flat case. It is associated with the quadratic form $f \mapsto\|\nabla f\|^{2}=\int_{\mathbb{R}^{d}}|\nabla f|^{2} e^{-\phi} \mathrm{d} x$ and defined by

$$
-\Delta_{\phi} f:=-\nabla_{\phi} \cdot \nabla f=-\Delta f+\nabla \phi \cdot \nabla f .
$$

The operator $-\Delta_{\phi}$ is nonnegative and symmetric.

For convenience, we shall also denote by $-\Delta_{\phi}$ the operator acting coordinate by coordinate on vector fields, that is for any smooth vector field $u,\left(\Delta_{\phi} u\right)_{i}=\Delta_{\phi} u_{i}$, and similarly extend it to matrices. In the same spirit, we introduce various differential operators. The formal adjoint of $D^{s}$ is defined by $\left(D^{s}\right)^{*} \mathfrak{F}=-D_{\phi}^{s} \cdot \mathfrak{F}$ for any matrix-valued function $\mathfrak{F}$, so that $D_{\phi}^{s}:=D^{s}-D^{s} \phi$ acts on matrix-valued functions and takes value in a space of vector fields. Here $D^{s} \phi \cdot \mathfrak{F}:=\nabla \phi \cdot \mathfrak{F}^{s}$. Let us consider

$$
-\Delta_{S} u:=-D_{\phi}^{s} \cdot D^{s} u \text { and } \quad-\Delta_{S \phi} u:=-D_{\phi}^{s} \cdot D^{s} u+(\nabla \phi \otimes \nabla \phi) u
$$

acting on a smooth vector field $u$. The differential operators $-\Delta_{S}$ and $-\Delta_{S \phi}$ are associated respectively with $\left\|D^{s} u\right\|^{2}$ and $\left\|D^{s} u\right\|^{2}+\|\nabla \phi \cdot u\|^{2}$, which appear in the various Korn and Poincaré-Korn inequalities. Additional details have been collected in Appendix B.2.

Theorem 3 (Associated operators acting on vector fields). Suppose (H1)-(H2)-(H3). Then the operators $-\Delta_{\phi},-\Delta_{S}$, and $-\Delta_{S \phi}$ are essentially self-adjoint on $L^{2}$. They have a common domain $\mathcal{D}$, finite dimensional kernels

$$
\operatorname{ker}\left(-\Delta_{\phi}\right)=\mathbb{R}^{d}, \quad \operatorname{ker}\left(-\Delta_{S}\right)=\mathbb{R}^{d} \oplus \mathcal{R}, \quad \operatorname{ker}\left(-\Delta_{S \phi}\right)=\mathcal{R}_{\phi},
$$

and positive spectral gaps. The spectral gap of $-\Delta_{\phi}$ is the Poincaré constant $C_{\mathrm{P}}$ while the spectral gaps of $-\Delta_{S}$ and $-\Delta_{S \phi}$ are estimated respectively in Theorems 1 and 2.

A positive spectral gap means that the infimum of the restriction of the spectrum to $(0,+\infty)$ is positive. Our last main result is devoted to a Korn-type inequality valid for vector fields $u \in L^{2}$ while Theorems 1 and 2 are limited to $u \in H^{1}$. We shall compose by inverse powers of the following positive operator

$$
\Lambda:=-\Delta_{\phi}+\mathrm{Id}
$$

acting on functions, vector fields or matrices, coordinate by coordinate. By Theorem $3, \Lambda$ is essentially selfadjoint (we keep the same name for the unique self-adjoint extension), $\Lambda \geq \mathrm{Id}$, and $\Lambda^{-1 / 2}$ is one-to-one from $H^{-1}$ into $L^{2}$ (see Propositions 7 and 8 for more details). In order to measure the possible non-axisymmetry of the potential $\phi$ in an $L^{2}$ setting, we introduce the rigidity of vector fields constant

$$
C_{\mathrm{RV0}}^{-1}:=\min _{A x+b \in\left(\mathcal{R}_{\phi}^{c} \oplus \mathbb{R}^{d}\right) \backslash\{0\}} \frac{\left\|\Lambda^{-1 / 2} \nabla \phi(x) \cdot(A x+b)\right\|^{2}}{\|A x+b\|^{2}} .
$$

when $\mathcal{R}_{\phi}^{c} \neq\{0\}$ and, by convention, $C_{\mathrm{RV} 0}:=0$ if $\mathcal{R}_{\phi}^{c}=\{0\}$. The proof that this constant $C_{\mathrm{Rvo}}$ is well-defined in $\mathbb{R}_{+}$is exactly similar to that for $C_{\mathrm{RV}}$.

Theorem 4 (Zeroth order Korn and Poincaré-Korn inequalities). Suppose (H1)-(H2)-(H3). Then there are a zeroth order Korn constant $C_{\mathrm{K} 0}$ and a zeroth order Poincaré-Korn constant $C_{\mathrm{PK} 0}$ with explicitly computable bounds depending only on $\phi$ such that, for all $u \in L^{2}$,

$$
\begin{aligned}
& \inf _{R \in \mathcal{R}}\left\|\Lambda^{-1 / 2} D(u-R)\right\|^{2}=\left\|\Lambda^{-1 / 2}(D u-\mathfrak{P}(D u))\right\|^{2} \leq C_{\mathrm{Ko}}\left\|\Lambda^{-1 / 2} D^{s} u\right\|^{2}, \\
& \inf _{R \in \mathcal{R}}\|u-\langle u\rangle-R\|^{2}=\|u-\langle u\rangle-\mathbb{P}(u)\|^{2} \leq C_{\mathrm{PK} 0}\left\|\Lambda^{-1 / 2} D^{s} u\right\|^{2} .
\end{aligned}
$$

As a consequence, there is a zeroth order precised Poincaré-Korn constant $C_{\mathrm{PK} 0}^{\prime}$ such that, for all $u \in L^{2}$,

$$
\inf _{R \in \mathcal{R}_{\phi}}\|u-R\|^{2}=\left\|u-\mathbb{P}_{\phi}(u)\right\|^{2} \leq C_{\mathrm{PKo}}^{\prime}\left\|\Lambda^{-1 / 2} D^{s} u\right\|^{2}+2 C_{\mathrm{RVo}}\left\|\Lambda^{-1 / 2} \nabla \phi \cdot u\right\|^{2} .
$$

Inequality (20) is a straightforward consequence of the Poincaré-Korn inequality (19) and the existence of the rigidity constant $C_{\mathrm{RV} 0}$. 
1.4. Main tools and considerations on the optimal cases and optimal constants. The paper relies on three main tools.

(1) Poincaré-Wirtinger and Poincaré-Lions inequalities. The proof of Theorems 1 and 2 for vector fields relies on Poincaré-Wirtinger inequalities for scalar functions, which go as follows.

Proposition 5. Assume that (H1), (H2) and (H3) hold, for some Poincaré constant $C_{\mathrm{P}}$. Then there exists a strong Poincaré constant $C_{\mathrm{SP}}>0$ such that

$$
\forall f \in H^{1}, \quad\|[\nabla \phi\rceil(f-\langle f\rangle)\|^{2} \leq C_{\mathrm{SP}}\|\nabla f\|^{2}
$$

with $C_{\mathrm{SP}} \leq C_{\phi}\left(1+C_{\mathrm{P}}\right)$. With $\Lambda$ as in (16), there exists also a Poincaré-Lions constant $C_{\mathrm{PL}}>0$ such that

$$
\forall f \in L^{2}, \quad\|f-\langle f\rangle\|^{2} \leq C_{\mathrm{PL}}\left\|\Lambda^{-1 / 2} \nabla f\right\|^{2} \leq C_{\mathrm{PL}}\|f-\langle f\rangle\|^{2} .
$$

Under the sole assumptions (H1)-(H2)-(H3), inequalities (21) and (22) are not completely standard. These inequalities are linked to the spectral properties of $-\Delta_{\phi}$, studied in Section 3, where elements of proofs of (21) and (22) are also collected. An estimate of $C_{\mathrm{PL}}$ is given in (41).

(2) The Schwarz Theorem allows us to write all components of the second-order differential of a vector field $u$ thanks to its symmetric components using the identity

$$
\forall i, j, k \in\{1, \cdots, d\}, \quad \partial_{k}\left(D^{a} u\right)_{i j}=\partial_{j}\left(D^{s} u\right)_{i k}-\partial_{i}\left(D^{s} u\right)_{j k}
$$

This algebraic property is at the core of all Korn-type inequalities, it means that derivatives of $D^{a} u$ are in the span of the derivatives of $D^{s} u$. Note that the Schwarz Theorem also implies $D^{a} \nabla=0$ which is central in the construction of the De Rham complex.

(3) The rigidity constants, as defined in (11), (12) and (17), measure the defects of axisymmetry of the potential $\phi$. See Appendix A.2 for a discussion.

Our method of proof can be summarised as follows: (i) we take care of the finite-dimensional parts $\mathcal{R}_{\phi}^{c}$ and $\mathfrak{M}_{\phi}^{c}$ thanks to the rigidity constants in (3), (ii) we apply twice the Poincaré inequality in (1), first in the form (21) and second in the form (22), so that we access second-order derivatives but remain at first order thanks to $\Lambda^{-1 / 2}$, (iii) we use the algebraic property in (2) to get rid of the derivatives of $D^{a} u$.

The infima in (8), (9), (13), (14), (19) and (20) are achieved respectively at $\mathfrak{P}(D u)=\left\langle D^{a} u\right\rangle$ (see Section 4.1), $\langle u\rangle+\mathbb{P}(u), \mathbb{P}_{\phi}(u), \mathfrak{P}_{\phi}(D u),\langle u\rangle+\mathbb{P}(u)$ and $\mathbb{P}_{\phi}(u)$ as a consequence of the definitions of the various orthogonal projections. In the Gaussian case (5), we have $D \mathbb{P}(u)=\mathfrak{P}(D u)$, but this relation is not true otherwise. The constants are estimated explicitely and a summary is provided in Appendix B.5.

1.5. A brief review of the literature and a conjecture. We refer to [19, Eq. (13)], [16, Chapter 3, Section 3.3], [8, page 291], and [24] for statements of the original Korn inequality which goes back to [28, 29, $30]$ in a bounded domain with Dirichlet conditions, and to $[32,6,5]$ for considerations on the best constant. There is a huge literature on applications to the Navier-Stokes equations and elasticity models, which is out of the scope of the present paper: see [34] for an introduction to Korn's inequality applied to these topics.

The case of Korn inequalities in bounded domains with Neumann boundary conditions was carried out in [12], driven by applications in kinetic theory in [13]. The proof relates the Korn constant to the so-called Grad number, which is further studied in [18] and related to other geometric bounds. The notion of Grad's number goes back to [20] in a bounded domain and was used in [12]. We refer to Appendix A.2 for a more detailed discussion and how it relates to our rigidity constants.

In bounded domains, inequalities of type (9) are usually called Poincaré-Korn estimates (see for instance [34, Section 1.3.1]), and inequality (14) is reminiscent of what is sometimes called the second Korn inequality: see [35, Inequality (7)] and [27, Theorem 2].

To our knowledge, the only result in the whole space with a confinement potential is [15, Section 5] where the Korn inequality (9) is proved by compactness, under an additional growth condition on $\nabla \phi$. The original contributions of this paper are

(i) a proof of weighted Poincaré-Korn and Korn inequalities, under rather general conditions,

(ii) a constructive method which provides us with quantitative estimates on the constants,

(iii) some optimal constants in the Gaussian case.

Our method is likely adaptable to bounded domains and also to fractional inequalities in the spirit of [33]. Inspired by the properties of the Gaussian Poincaré inequality, e.g., in [10], we finally make the following conjecture. 
Conjecture (Optimal constants). For a given $\phi$ satisfying $(\mathrm{H} 1)-(\mathrm{H} 2)-(\mathrm{H} 3)$ with $\int_{\mathbb{R}^{d}} x_{i} x_{j} e^{-\phi(x)} \mathrm{d} x=\delta_{i j}$ for all $i, j \in\{1, \cdots, d\}$ and $D^{2} \phi \geq \mathrm{Id}$, one has $C_{\mathrm{PK}} \geq 2$ and $C_{\mathrm{K}} \geq 4$, with equality in the normalized centred Gaussian case (5) and only in that case.

1.6. Outline of the paper. In Section 2 we prove Theorem 1 in the simple case of Gaussian potentials. This has a pedagogical interest but also an interest per se as the method captures some (conjectured) optimal constants. Section 3 is devoted to classical results on the Witten-Laplace operator on functions and a sketch of the proof of Poincaré inequalities under assumptions (H1)-(H2)-(H3) with some short quantitative proofs for which we lack of references. In Section 4 we prove Theorems 1 and 2 in the general case. Section 5 is devoted to the functional analysis of operators (Theorem 3) associated with various quadratic forms under consideration. We prove Theorem 4 on zeroth order Korn inequalities in Section 6. Appendix A is devoted to generalizations, a discussion of the measure of the defects of axisymmetry by rigidity constants, and an elementary application of our main results to a simple kinetic equation with multiple conservations laws. For the convenience of the reader, some computational details are collected in Appendix B.

\section{Proof of the Korn inequalities of Theorem 1 in the Gaussian case}

Inspired by the proof of (1), we first prove inequalities (8) and (9) of Theorem 1 for the normalized Gaussian measure, and establish the optimality of the constants in that case. We begin with two useful identities valid for a general function $\phi \in \mathrm{W}_{\text {loc }}^{2, \infty}\left(\mathbb{R}^{d}\right)$ and any $u \in C_{c}^{1}\left(\mathbb{R}^{d} ; \mathbb{R}^{d}\right)$,

$$
\begin{aligned}
& \left\|D^{a} u\right\|^{2}+\|(\nabla-\nabla \phi) \cdot u\|^{2}=\left\|D^{s} u\right\|^{2}+\int_{\mathbb{R}^{d}} D^{2} \phi: u \otimes u e^{-\phi} \mathrm{d} x, \\
& \|D u\|^{2} \leq 2\left\|D^{s} u\right\|^{2}+\int_{\mathbb{R}^{d}} D^{2} \phi: u \otimes u e^{-\phi} \mathrm{d} x .
\end{aligned}
$$

Identity (24) is obtained by a simple integration by parts, a commutation and the Schwarz Theorem (or (2) integrated against $e^{-\phi}$ ), while (25) follows from $|D u|^{2}=\left|D^{s} u\right|^{2}+\left|D^{a} u\right|^{2}$.

In the remainder of this section, let us focus on the Gaussian case (5) such that

$$
e^{-\phi(x)}=(2 \pi)^{-d / 2} e^{-\frac{1}{2}|x|^{2}}
$$

is the standard centred normalized Gaussian. This is the only Gaussian function satisfying hypotheses (H1) with the additional normalization $\left\langle D^{2} \phi\right\rangle=\mathrm{Id}$, and it satisfies (H2) with $\varepsilon=0$ and $C_{\varepsilon}=C_{0}=d$ and it satisfies (H3) with $C_{\mathrm{P}}=1$. We first recall the following improved version of the Poincaré inequality.

Lemma 6 (Improved Poincaré inequality). Assume (5). Then for any $u \in H^{1}$ such that $\left\langle u_{i} x_{j}\right\rangle=\left\langle u_{i}\right\rangle=0$ with $i, j=1, \ldots, d$, there holds

$$
2\|u\|^{2} \leq\|D u\|^{2} .
$$

This result is standard: the operator $-\Delta_{\phi}$ reduces after conjugation by $e^{-\phi / 2}$ to the harmonic oscillator $P_{\phi}=-\Delta+|x|^{2} / 4-d / 2$ which has a discrete spectrum made of all nonnegative integers (see Section 3 for the definition of the operator). The lowest eigenvalue is 0 with multiplicity 1 and the first positive eigenvalue is 1 with multiplicity $d$ and eigenfunctions $x_{j}, j=1,2, \ldots, d$. Conditions on $u$ amount to the orthogonality condition to these two eigenspaces, so that 2 corresponds to the next eigenvalue. The result follows from the spectral theorem (see for instance to [37, Lemma 2 of Chapter V and Chapter 8] or [11]).

Proof of Theorem 1 in the Gaussian case. Since $D^{s}\left(\left\langle D^{a} u\right\rangle x\right)=D^{s}(\langle u\rangle)=D(\langle u\rangle)=0$ and $\mathbb{P}\left(\left\langle D^{a} u\right\rangle x\right)=$ $\left\langle D^{a} u\right\rangle x$, it is enough to prove the inequalities for a vector field $u \in H^{1}$ such that $\left\langle D^{a} u\right\rangle=0$ and $\langle u\rangle=0$. We have to show that

$$
\|u\|^{2} \leq 2\left\|D^{s} u\right\|^{2} \quad \text { and } \quad\|D u\|^{2} \leq 4\left\|D^{s} u\right\|^{2} .
$$

Let us define the corrected vector field $v \in H^{1}$ by

$$
v(x):=u(x)-B x \quad \text { where } \quad B_{i j}:=\left\langle u_{i} x_{j}\right\rangle,
$$

and note the elementary property (using that $e^{-\phi} \mathrm{d} x$ is Gaussian)

$$
B_{i j}=\left\langle u_{i} x_{j}\right\rangle=\int_{\mathbb{R}^{d}} u_{i} x_{j} e^{-\phi} \mathrm{d} x=\int_{\mathbb{R}^{d}} \partial_{j} u_{i} e^{-\phi} \mathrm{d} x=\langle D u\rangle_{i j} .
$$


This implies that the matrix $B$ is symmetric since $\left\langle D^{a} u\right\rangle=0$ and that $v$ satisfies $\left\langle v_{i} x_{j}\right\rangle=\left\langle v_{i}\right\rangle=0$ for all $i, j=1, \ldots, d$. We can then apply the improved Poincaré inequality (26) in Lemma 6 to $v$ and get

$$
2\|v\|^{2} \leq\|D v\|^{2} .
$$

Using this together with (25) and $D^{2} \phi=\mathrm{Id}$, we obtain $2\|v\|^{2} \leq\|D v\|^{2} \leq 2\left\|D^{s} v\right\|^{2}+\|v\|^{2}$ which implies $\|v\|^{2} \leq 2\left\|D^{s} v\right\|^{2}$ and $\|D v\|^{2} \leq 4\left\|D^{s} v\right\|^{2}$, i.e., (27) written for $v$. Next we compute

$$
\begin{aligned}
& \|D u\|^{2}=\|D v+B\|^{2}=\left\|D^{s} v+B\right\|^{2}+\left\|D^{a} v\right\|^{2}=\|D v\|^{2}+|B|^{2}+2 \int_{\mathbb{R}^{d}}\left(D^{s} v: B\right) e^{-\phi} \mathrm{d} x, \\
& \left\|D^{s} u\right\|^{2}=\left\|D^{s} v+B\right\|^{2}=\left\|D^{s} v\right\|^{2}+|B|^{2}+2 \int_{\mathbb{R}^{d}}\left(D^{s} v: B\right) e^{-\phi} \mathrm{d} x, \\
& \|u\|^{2}=\|v+B x\|^{2}=\|v\|^{2}+|B|^{2}+2 \int_{\mathbb{R}^{d}}(v \cdot B x) e^{-\phi} \mathrm{d} x=\|v\|^{2}+|B|^{2},
\end{aligned}
$$

where we used the fact that $\left(D^{s} u+B\right)$ and $D^{a} u$ are orthogonal in $L^{2}$ and $\left\langle v_{i} x_{j}\right\rangle=0$. By an integration by parts, we obtain

$$
\int_{\mathbb{R}^{d}}\left(D^{s} v: B\right) e^{-\phi} \mathrm{d} x=\frac{1}{2} \sum_{i, j} B_{i j} \int_{\mathbb{R}^{d}}\left(\partial_{i} v_{j}+\partial_{j} v_{i}\right) e^{-\phi} \mathrm{d} x=\frac{1}{2} \sum_{i, j} B_{i j} \int_{\mathbb{R}^{d}}\left(x_{i} v_{j}+x_{j} v_{i}\right) e^{-\phi} \mathrm{d} x=0
$$

using again $\left\langle v_{i} x_{j}\right\rangle=0$. Altogether, we deduce that

$$
\|D u\|^{2}=\|D v\|^{2}+|B|^{2}, \quad\left\|D^{s} u\right\|^{2}=\left\|D^{s} v\right\|^{2}+|B|^{2}, \quad\|u\|^{2}=\|v\|^{2}+|B|^{2} .
$$

We deduce (27) on $u$ from (27) on $v$ and the last equations, which proves (8) with $C_{\mathrm{K}} \leq 4$ and (9) with $C_{\mathrm{PK}} \leq 2$. To saturate (27) it is enough to search for $u=v$ with $B=0$. With $u(x)=\left(1-x_{2}^{2}, x_{1} x_{2}, 0, \ldots, 0\right)^{\perp}$, an elementary computation (see details in Appendix B.3) shows that

$$
\langle u\rangle=0, \quad\|u\|^{2}=3, \quad \mathbb{P}(u)=0, \quad \mathfrak{P}(D u)=0=\left\langle D^{a} u\right\rangle, \quad\left\|D^{s} u\right\|^{2}=\frac{3}{2}, \quad\left\|D^{a} u\right\|^{2}=\frac{9}{2}, \quad\|D u\|^{2}=6 .
$$

This completes the proof of (8) with $C_{\mathrm{K}}=4$ and (9) with $C_{\mathrm{PK}}=2$.

It remains to establish (10). By expanding the square $\int_{\mathbb{R}^{d}}\left|D\left(u e^{-\phi / 2}\right)\right|^{2} \mathrm{~d} x$ as in [14, ineq. (4)], we obtain after one integration by parts that

$$
\int_{\mathbb{R}^{d}}|x|^{2}|u(x)|^{2} e^{-\phi(x)} \mathrm{d} x \leq 4 \int_{\mathbb{R}^{d}}|D u|^{2} e^{-\phi} \mathrm{d} x+2 d \int_{\mathbb{R}^{d}}|u|^{2} e^{-\phi} \mathrm{d} x .
$$

Combined with (8) and (9), this completes the proof with $C_{\mathrm{PK}} \leq C_{\mathrm{SPK}} \leq 2(2 d+9)$.

\section{The Witten-Laplace operator on scalar functions and Poincaré inequalities}

Here we consider the Poincaré inequalities of Proposition 5 and some related properties of the WittenLaplace operator $\Delta_{\phi}$, as defined in (15), in the case of a general probability measure $e^{-\phi} \mathrm{d} x$ with a potential $\phi$ such that assumptions (H1)-(H2)-(H3) are fulfilled. Some results of this section are classical and we claim no originality. For a general theory of self-adjoint operators, we refer for instance to [38, 37] and we refer to $[40,22,39,25]$ or [21] for more details on Witten-Laplace operators. Proofs are given when we are not aware of any precise reference or when we look for explicit estimates.

3.1. Two toolboxes and the proof of the strong Poincaré inequality (Proposition 5). For all functions $f, g \in \mathcal{C}_{c}^{\infty}\left(\mathbb{R}^{d} ; \mathbb{R}\right)$, we have by integration by parts

$$
\left(-\Delta_{\phi} f, g\right)=-\left(\nabla_{\phi} \cdot \nabla f, g\right)=(\nabla f, \nabla g),
$$

so that $-\nabla_{\phi}=-\nabla+\nabla \phi$ is the formal adjoint of $\nabla,-\Delta_{\phi}$ is nonnegative and symmetric, and $\Lambda$, as defined by (16), is symmetric. The Lax-Milgram theorem allows us to solve in $H^{1}$, equipped with the norm $f \mapsto\left(\|f\|^{2}+\|\nabla f\|^{2}\right)^{1 / 2}$, the problem $\Lambda f=\xi$ for any given $\xi \in H^{-1}$, and to build a self-adjoint extension of $\Lambda$ associated to the coercive bilinear form $(f, g) \mapsto(\nabla f, \nabla g)+(f, g)$. On the other hand, by the well-known change of function $f \mapsto e^{-\phi / 2} f, \Lambda$ is conjugated to

$$
P_{\phi}:=e^{-\phi / 2} \Lambda e^{-\phi / 2}=-\Delta+\frac{1}{4}|\nabla \phi|^{2}-\frac{1}{2} \Delta \phi+1
$$

acting on the usual space $L^{2}(\mathrm{~d} x)$. From (H2), we get that $|\nabla \phi|^{2} / 4-\Delta \phi / 2$ is bounded from below. From Kato's result [26] (also see, e.g., [37, Theorem X-28]), this implies that $\Lambda$ has a unique Friedrichs self-adjoint 
extension such that $\mathcal{C}_{c}^{\infty}\left(\mathbb{R}^{d} ; \mathbb{R}\right)$ is dense in its domain w.r.t. the graph norm, that is, $\Lambda$ is essentially selfadjoint. For notational simplicity, we use the same name for the operator and for its extension. We denote by $\mathcal{D}(\Lambda)$ the domain of $\Lambda$.

Hence $\mathcal{C}_{c}^{\infty}\left(\mathbb{R}^{d} ; \mathbb{R}\right)$ is a core for the self-adjoint operator $\Lambda \geq \mathrm{Id}$, which has a one-to-one operator extension from $H^{1}$ to $H^{-1}$. Tools of functional calculus and spectral analysis apply. This gives sense to $\Lambda^{\sigma}$ with domain $\mathcal{D}\left(\Lambda^{\sigma}\right)$ for all $\sigma \in \mathbb{R}$. For instance, $\mathcal{D}\left(\Lambda^{1 / 2}\right)=H^{1}$ and $\Lambda^{1 / 2}$ has a bounded one-to-one operator extension from $L^{2}$ to $H^{-1}$ by duality. Recall that no specific growth, apart from the general condition (H2), is assumed on $|\nabla \phi|$ at infinity in the computations of this section (see [23,21, 17] for other results without growth condition). Let us show that (H2) implies that $\lfloor\nabla \phi\rceil f$ is square integrable whenever $f \in H^{1}$, which allows to make sense of $\|\nabla \phi \cdot u\|$ in inequalities (13) and (14).

Proposition 7 ( $H^{1}$ toolbox). Assume (H2). Then the space $H^{1}$ is

$$
H^{1}=\left\{f \in L^{2}: \nabla f \in L^{2} \text { and }\lfloor\nabla \phi\rceil f \in L^{2}\right\}
$$

and for any $f \in L^{2}$, we have the inequalities

$$
\begin{aligned}
& \left\|\nabla \Lambda^{-1 / 2} f\right\|^{2} \leq\|f\|^{2}, \quad\left\|\lfloor\nabla \phi\rceil \Lambda^{-1 / 2} f\right\|^{2} \leq C_{\phi}\|f\|^{2}, \\
& \left\|\Lambda^{-1 / 2} \nabla f\right\|^{2} \leq\|f\|^{2}, \quad\left\|\Lambda^{-1 / 2}\lfloor\nabla \phi\rceil f\right\|^{2} \leq C_{\phi}\|f\|^{2} .
\end{aligned}
$$

Proof. For all $f \in D(\Lambda)$ we have $\|\nabla f\|^{2} \leq(\Lambda f, f)=\left\|\Lambda^{1 / 2} f\right\|^{2}$. By density of $D(\Lambda)$ in $H^{1}$ we get $\|\nabla f\|^{2} \leq\left\|\Lambda^{1 / 2} f\right\|^{2}$ for all $f \in H^{1}$ and applying this inequality to $\Lambda^{-1 / 2} f \in H^{1}$ proves the first inequality in (28).

Let us note that $0 \leq|\nabla \phi|^{2}-4 \sqrt{d}\left|D^{2} \phi\right|+C_{\phi}-1 \leq|\nabla \phi|^{2}-4 \Delta \phi+C_{\phi}-1$ because $\Delta \phi \leq \sqrt{d}\left|D^{2} \phi\right|$ and according to (6), so that

$$
\lfloor\nabla \phi\rceil^{2} \leq 8\left(\frac{1}{4}|\nabla \phi|^{2}-\frac{1}{2} \Delta \phi\right)+C_{\phi}
$$

As a consequence, we get the operator inequality $\lfloor\nabla \phi\rceil^{2} \leq-8 \Delta_{\phi}+C_{\phi}$ Id $\leq C_{\phi} \Lambda$ using the fact that the usual Laplacian $-\Delta$ is nonnegative on $L^{2}(\mathrm{~d} x)$ and $C_{\phi} \geq 8$. This implies that, for all $f \in D(\Lambda)$, we have that $\lfloor\nabla \phi\rceil f$ is in $L^{2}$ and $\|\lfloor\nabla \phi\rceil f\|^{2} \leq C_{\phi}(\Lambda f, f)=C_{\phi}\left\|\Lambda^{1 / 2} f\right\|^{2}$. By density of $D(\Lambda)$ in $H^{1}$, we get

$$
\forall f \in H^{1}, \quad\|\lfloor\nabla \phi\rceil f\|^{2} \leq C_{\phi}\left\|\Lambda^{1 / 2} f\right\|^{2} .
$$

For any $f \in L^{2}$, applying (30) to $\Lambda^{-1 / 2} f \in H^{1}$ gives the second inequality in (28). Inequalities in (29) are obtained from (28) by considering the adjoint operators.

Proof of the strong Poincaré inequality (21). So far we did not use (H3) and its spectral consequences. Using the density of $\mathcal{C}_{c}^{\infty}\left(\mathbb{R}^{d}, \mathbb{R}\right)$ in $D(\Lambda)$ and (H3), we get that 0 is an isolated eigenvalue of $-\Delta_{\phi}=\Lambda-1$ with associated eigenspace $\mathbb{R}$. Inequality (21) follows from (30) applied to $f-\langle f\rangle$ and (H3), with $C_{\mathrm{SP}} \leq$ $C_{\phi}\left(1+C_{\mathrm{P}}\right)$.

The following toolbox is a key step in proof of the Poincaré-Lions inequality (22).

Proposition $8(D(\Lambda)$-Toolbox). Assume (H1) and (H2). Then

$$
\mathcal{D}(\Lambda)=\left\{f \in L^{2}:\left\|\lfloor\nabla \phi\rceil^{2} f\right\|+\|\lfloor\nabla \phi\rceil \nabla f\|+\left\|D^{2} f\right\|<+\infty\right\}
$$

and there exists a positive constant $C_{\mathrm{B}}$ depending only on $C_{\phi}, C_{\phi}^{\prime}$ and $d$ such that, for any $f \in L^{2}$,

$$
\begin{aligned}
& \left\|D^{2} \Lambda^{-1} f\right\|^{2}+\left\|\lfloor\nabla \phi\rceil \nabla \Lambda^{-1} f\right\|^{2}+\left\|\lfloor\nabla \phi\rceil^{2} \Lambda^{-1} f\right\|^{2} \leq C_{\mathrm{B}}\|f\|^{2}, \\
& \left\|\Lambda^{-1} D^{2} f\right\|^{2}+\left\|\Lambda^{-1}\lfloor\nabla \phi\rceil \nabla f\right\|^{2}+\left\|\Lambda^{-1}\lfloor\nabla \phi\rceil^{2} f\right\|^{2} \leq C_{\mathrm{B}}\|f\|^{2} .
\end{aligned}
$$

Proof. Inequality (32) follows from (31) by duality using (H2).

Let us denote by $\mathcal{S}$ the subspace of $L^{2}$ such that $D^{2} f,\lfloor\nabla \phi\rceil \nabla f$ and $\lfloor\nabla \phi\rceil^{2} f$ are square integrable. It is elementary to check that $\mathcal{D}(\Lambda) \subset \mathcal{S}$. In order to prove that, reciprocally, $\mathcal{S} \subset \mathcal{D}(\Lambda)$, let us argue by density of $\mathcal{C}_{c}^{\infty}\left(\mathbb{R}^{d} ; \mathbb{R}\right)$. For any $f \in \mathcal{C}_{c}^{\infty}\left(\mathbb{R}^{d} ; \mathbb{R}\right)$, let us prove that $\xi=\Lambda f=-\Delta_{\phi} f+f$ is such that

$$
\left\|\lfloor\nabla \phi\rceil^{2} f\right\|^{2}+\|\lfloor\nabla \phi\rceil \nabla f\|^{2}+\left\|D^{2} f\right\|^{2} \leq C_{\mathrm{B}}\|\xi\|^{2}
$$

for some explicit constant $C_{\mathrm{B}}$, so that $\mathcal{D}(\Lambda)=\mathcal{S}$ and (31) directly follow.

It follows from $\|f\|^{2} \leq(\Lambda f, f) \leq\|\Lambda f\|\|f\|$ that $\|f\| \leq\|\xi\|$. Similarly, using (28), we have

$$
\|\lfloor\nabla \phi\rceil f\| \leq C_{\phi}^{1 / 2}\left\|\Lambda^{1 / 2} f\right\|=C_{\phi}^{1 / 2}(\Lambda f, f)^{1 / 2} \leq C_{\phi}^{1 / 2}\|\xi\| .
$$


Next, we estimate $\left\|\lfloor\nabla \phi\rceil^{2} f\right\|$. Using (28) and the triangular inequality we have

$$
\left\|\lfloor\nabla \phi\rceil^{2} f\right\|=\|\lfloor\nabla \phi\rceil\lfloor\nabla \phi\rceil f\| \leq C_{\phi}^{1 / 2}\left\|\Lambda^{1 / 2}(\lfloor\nabla \phi\rceil f)\right\| \leq C_{\phi}^{1 / 2}\|\nabla(\lfloor\nabla \phi\rceil f)\|+C_{\phi}^{1 / 2}\|\lfloor\nabla \phi\rceil f\| .
$$

With $\nabla(\lfloor\nabla \phi\rceil f)=\lfloor\nabla \phi\rceil \nabla f+(\nabla\lfloor\nabla \phi\rceil) f$, we get

$$
\begin{aligned}
\left\|\lfloor\nabla \phi\rceil^{2} f\right\| & \leq C_{\phi}^{1 / 2}\|\lfloor\nabla \phi\rceil \nabla f\|+C_{\phi}^{1 / 2}\|\lfloor\nabla \phi\rceil f\|+C_{\phi}^{1 / 2}\|(\nabla\lfloor\nabla \phi\rceil) f\| \\
& \leq C_{\phi}^{1 / 2}\|\lfloor\nabla \phi\rceil \nabla f\|+C_{\phi}\|\xi\|+C_{\phi}^{1 / 2}\|(\nabla\lfloor\nabla \phi\rceil) f\|
\end{aligned}
$$

by (34). Estimate (6) yields

$$
|\nabla\lfloor\nabla \phi\rceil| \leq \frac{\left|D^{2} \phi\right||\nabla \phi|}{\lfloor\nabla \phi\rceil} \leq \frac{1}{4} C_{\phi}^{-1 / 2}\lfloor\nabla \phi\rceil^{2}+\frac{1}{4} C_{\phi}^{\prime} C_{\phi}^{-1 / 2}
$$

and, as a consequence,

$$
\left\|\lfloor\nabla \phi\rceil^{2} f\right\| \leq C_{\phi}^{1 / 2}\|\lfloor\nabla \phi\rceil \nabla f\|+C_{\phi}\|\xi\|+\frac{1}{4}\left\|\lfloor\nabla \phi\rceil^{2} f\right\|+\frac{1}{4} C_{\phi}^{\prime}\|f\|,
$$

so that using $C_{\phi}^{\prime} \geq C_{\phi}$ and $\|f\| \leq\|\xi\|$, we get

$$
\left\|\lfloor\nabla \phi\rceil^{2} f\right\| \leq \frac{4}{3} C_{\phi}^{1 / 2}\|\lfloor\nabla \phi\rceil \nabla f\|+\frac{5}{3} C_{\phi}^{\prime}\|\xi\| .
$$

Next we estimate $\|\lfloor\nabla \phi\rceil \nabla f\|^{2}$ by

$$
\begin{aligned}
\|\lfloor\nabla \phi\rceil \nabla f\|^{2} & =\left(\lfloor\nabla \phi\rceil^{2} \nabla f, \nabla f\right)=\left(\nabla\left(\lfloor\nabla \phi\rceil^{2} f\right), \nabla f\right)-\left(\left(\nabla\lfloor\nabla \phi\rceil^{2}\right) f, \nabla f\right) \\
& =\left(\lfloor\nabla \phi\rceil^{2} f, \xi-f\right)-\left(\left(\nabla\lfloor\nabla \phi\rceil^{2}\right) f, \nabla f\right) \\
& \leq\left(\lfloor\nabla \phi\rceil^{2} f, \xi\right)-2((\nabla\lfloor\nabla \phi\rceil) f,\lfloor\nabla \phi\rceil \nabla f) \\
& \leq\left\|\lfloor\nabla \phi\rceil^{2} f\right\|\|\xi\|+2\|(\nabla\lfloor\nabla \phi\rceil) f\|\|\lfloor\nabla \phi\rceil \nabla f\| .
\end{aligned}
$$

Using (35) and $\|f\| \leq\|\xi\|$, we get

$$
\|\lfloor\nabla \phi\rceil \nabla f\|^{2} \leq\left\|\lfloor\nabla \phi\rceil^{2} f\right\|\|\xi\|+\frac{1}{2} C_{\phi}^{-1 / 2}\left\|\lfloor\nabla \phi\rceil^{2} f\right\|\|\lfloor\nabla \phi\rceil \nabla f\|+\frac{1}{2} C_{\phi}^{\prime} C_{\phi}^{-1 / 2}\|\xi\|\|\lfloor\nabla \phi\rceil \nabla f\| .
$$

With elementary estimates, we deduce from (36)-(37) that

$$
\|\lfloor\nabla \phi\rceil \nabla f\| \leq 9 C_{\phi}^{\prime}\|\xi\| \quad \text { and } \quad\left\|\lfloor\nabla \phi\rceil^{2} f\right\| \leq 14 C_{\phi}^{\prime}\|\xi\| .
$$

Integrations by parts show that

$$
\begin{aligned}
\left\|D^{2} f\right\|^{2} & =\sum_{i, j}\left(\partial_{i j} f, \partial_{i j} f\right)=\sum_{i, j}\left(\partial_{j} f,-\partial_{i i j} f+\partial_{i j} f \partial_{i} \phi\right) \\
& =\sum_{i, j}\left(\partial_{j} f, \partial_{j}\left(-\partial_{i i} f\right)\right)+\frac{1}{2} \sum_{i, j}\left(\partial_{i}\left(\left|\partial_{j} f\right|^{2}\right), \partial_{i} \phi\right)=(\nabla f, \nabla(-\Delta f))+\frac{1}{2}\left(|\nabla f|^{2},|\nabla \phi|^{2}-\Delta \phi\right) .
\end{aligned}
$$

Using the elementary estimates

$$
\begin{aligned}
& (\nabla f, \nabla(-\Delta f))=\left(\Delta_{\phi} f, \Delta f\right)=(f-\xi, \Delta f) \leq(f, \Delta f)+\|\xi\|\|\Delta f\|, \\
& (f, \Delta f)=\left(f, \Delta_{\phi} f\right)+(f, \nabla \phi \cdot \nabla f)=-\|\nabla f\|^{2}+\frac{1}{2}\left(\nabla f^{2}, \nabla \phi\right) \leq-\frac{1}{2}\left(f^{2}, \Delta_{\phi} \phi\right)=\frac{1}{2}\left(|f|^{2},|\nabla \phi|^{2}-\Delta \phi\right),
\end{aligned}
$$

and using (6) and $\lfloor\nabla \phi\rceil \geq 1$ and the fact that

$|\nabla \phi|^{2}-\Delta \phi \leq|\nabla \phi|^{2}+\sqrt{d}\left|D^{2} \phi\right| \leq|\nabla \phi|^{2}+\frac{1}{4}\left(|\nabla \phi|^{2}+C_{\phi}-1\right) \leq \frac{1}{4}\left(5\lfloor\nabla \phi\rceil^{2}+C_{\phi}-6\right) \leq \frac{1}{4}\left(C_{\phi}-1\right)\lfloor\nabla \phi\rceil$, we obtain, using also (38), the estimate

$$
\begin{gathered}
\frac{1}{d}\|\Delta f\|^{2} \leq\left\|D^{2} f\right\|^{2} \leq\|\xi\|\|\Delta f\|+\frac{1}{2}\left(|f|^{2}+|\nabla f|^{2},|\nabla \phi|^{2}-\Delta \phi\right) \\
\leq\|\xi\|\|\Delta f\|+\frac{1}{8}\left(C_{\phi}-1\right)\left(\left\|\lfloor\nabla \phi\rceil^{2} f\right\|^{2}+\|\lfloor\nabla \phi\rceil \nabla f\|^{2}\right) \leq\|\xi\|\|\Delta f\|+C\|\xi\|^{2}
\end{gathered}
$$

with $C=\frac{1}{8} 277\left(C_{\phi}-1\right) C_{\phi}^{\prime 2}$ because $277=9^{2}+14^{2}$. As a straightforward consequence, we obtain

$$
\|\Delta f\| \leq \frac{1}{2}\left(d+\sqrt{d^{2}+4 C}\right)\|\xi\| \quad \text { and } \quad\left\|D^{2} f\right\|^{2} \leq \frac{C}{d}+\frac{1}{2}\left(d+\sqrt{d^{2}+4 C}\right)\|\xi\|^{2} .
$$

With (38), this completes the proof of (33). A detailed computation of $C_{\mathrm{B}}$ is given in Appendix B.4. 
3.2. The Poincaré-Lions inequality (Proposition 5). We now focus on (22). As a preliminary remark, note that this inequality is the counterpart in the whole space of the so-called Lions lemma in the smooth bounded domain case $\Omega \subset \mathbb{R}^{d}$, which amounts to the existence of some $c_{\Omega}>0$ such that

$$
\forall f \in L^{2}(\Omega), \quad c_{\Omega}\|f-\langle f\rangle\|_{L^{2}(\Omega)}^{2} \leq\|\nabla f\|_{H^{-1}(\Omega)}^{2} \leq d\|f-\langle f\rangle\|_{L^{2}(\Omega)}^{2}
$$

(see for instance [16] and [9, Theorem 6.11.4]). This inequality belongs to the folklore in Hodge theory, see for instance [22] or [25], with variants involving the so-called Witten-Laplacian on one-forms.

Proof of the Poincaré-Lions inequality (22). First note that the right inequality directly follows from (29) applied to $f-\langle f\rangle$. We focus on the left one. The spectral theorem implies for all $f \in D(\Lambda)$ with $\langle f\rangle=0$

$$
\left(1+C_{\mathrm{P}}\right)^{-1}\|f\|^{2} \leq\left(\left(-\Delta_{\phi}\right) \Lambda^{-1} f, f\right)=\left(\Lambda^{1 / 2} \nabla \Lambda^{-1} f, \Lambda^{-1 / 2} \nabla f\right) \leq\left\|\Lambda^{1 / 2} \nabla \Lambda^{-1} f\right\|\left\|\Lambda^{-1 / 2} \nabla f\right\|
$$

because $1 /\left(1+C_{\mathrm{P}}\right) \leq s /(s+1)$ for any $s \in\left[1 / C_{\mathrm{P}}, \infty\right)$. Let us prove that $\Lambda^{1 / 2} \nabla \Lambda^{-1}$ is a bounded operator. Using the commutator $[\Lambda, \nabla]=-D^{2} \phi \nabla$, we compute

$$
\begin{aligned}
\Lambda^{1 / 2} \nabla \Lambda^{-1} & =\Lambda^{-1 / 2} \Lambda \nabla \Lambda^{-1}=\Lambda^{-1 / 2} \nabla+\Lambda^{-1 / 2}[\Lambda, \nabla] \Lambda^{-1} \\
& =\Lambda^{-1 / 2} \nabla-\Lambda^{-1 / 2} D^{2} \phi \nabla \Lambda^{-1}=\Lambda^{-1 / 2} \nabla+\Lambda^{-1 / 2}\lfloor\nabla \phi\rceil\left(\lfloor\nabla \phi\rceil^{-1} D^{2} \phi\lfloor\nabla \phi\rceil^{-1}\right)\lfloor\nabla \phi\rceil \nabla \Lambda^{-1} .
\end{aligned}
$$

From (29), we know that $\Lambda^{-1 / 2} \nabla$ and $\Lambda^{-1 / 2}\lfloor\nabla \phi\rceil$ are bounded respectively by 1 and $\sqrt{C_{\phi}}$, from (31) the operator $\lfloor\nabla \phi\rceil \nabla \Lambda^{-1}$ is bounded by $\sqrt{C_{\mathrm{B}}}$, and we have

$$
\lfloor\nabla \phi\rceil^{-1} D^{2} \phi\lfloor\nabla \phi\rceil^{-1} \leq \frac{C_{\phi}^{\prime}}{4 \sqrt{C_{\phi}}}
$$

as a consequence of (6). Altogether, $\Lambda^{1 / 2} \nabla \Lambda^{-1}$ is bounded and

$$
\left\|\Lambda^{1 / 2} \nabla \Lambda^{-1} f\right\| \leq\left(1+\frac{1}{4} C_{\phi}^{\prime} \sqrt{C_{\mathrm{B}}}\right)\|f\|,
$$

which completes the proof with

$$
C_{\mathrm{PL}}=\left(1+C_{\mathrm{P}}\right)^{2}\left(1+\frac{1}{4} C_{\phi}^{\prime} \sqrt{C_{\mathrm{B}}}\right)^{2} .
$$

\section{Proof of the Korn inequalities of Theorems 1 and 2 for general potentials}

In this section, we assume that the potential satisfies (H1), (H2) and (H3).

4.1. Proof of Theorem 1. As a preliminary remark, we recall that

$$
\forall u \in H^{1}, \quad \mathfrak{P}(D u)=\left\langle D^{a} u\right\rangle .
$$

Indeed $D u=\left(D u-\left\langle D^{a} u\right\rangle\right)+\left\langle D^{a} u\right\rangle$ is an orthogonal decomposition because

$$
\left(D u-\left\langle D^{a} u\right\rangle,\left\langle D^{a} u\right\rangle\right)=\left(D^{a} u-\left\langle D^{a} u\right\rangle,\left\langle D^{a} u\right\rangle\right)+\left(D^{s} u,\left\langle D^{a} u\right\rangle\right)=\left\langle D^{s} u\right\rangle:\left\langle D^{a} u\right\rangle=0
$$

and the uniqueness of this decomposition shows the result.

$\triangleright$ Proof of (8). Let us take $u \in H^{1}$ such that $\langle u\rangle=0$ and $\left\langle D^{a} u\right\rangle=0$. Using the Poincaré-Lions inequality (22), we have

$$
\|D u\|^{2}=\left\|D^{s} u\right\|^{2}+\left\|D^{a} u\right\|^{2}=\left\|D^{s} u\right\|^{2}+\sum_{i, j=1}^{d}\left\|\left(D^{a} u\right)_{i j}\right\|^{2} \leq\left\|D^{s} u\right\|^{2}+C_{\mathrm{PL}}\left\|\Lambda^{-1 / 2} \nabla\left(D^{a} u\right)\right\|^{2}
$$

with $\left\|\Lambda^{-1 / 2} \nabla\left(D^{a} u\right)\right\|^{2}=\sum_{i, j=1}^{d}\left\|\Lambda^{-1 / 2} \nabla\left(D^{a} u\right)_{i j}\right\|^{2}$. The Schwarz Theorem as stated in (23) gives

$$
\left\|\Lambda^{-1 / 2} \nabla\left(D^{a} u\right)\right\|^{2} \leq 2 \sum_{i, j, k=1}^{d}\left(\left\|\Lambda^{-1 / 2} \partial_{i}\left(D^{s} u\right)_{j k}\right\|^{2}+\left\|\Lambda^{-1 / 2} \partial_{j}\left(D^{s} u\right)_{i k}\right\|^{2}\right)=4 \sum_{j, k=1}^{d}\left\|\Lambda^{-1 / 2} \nabla\left(D^{s} u\right)_{j k}\right\|^{2} .
$$

The right-hand side of the Poincaré-Lions inequality (22) yields

$$
\sum_{j, k=1}^{d}\left\|\Lambda^{-1 / 2} \nabla\left(D^{s} u\right)_{j k}\right\|^{2} \leq \sum_{j, k=1}^{d}\left\|\left(D^{s} u\right)_{j k}\right\|^{2}=\left\|D^{s} u\right\|^{2}
$$


Together with (42) and (43), this gives $\|D u\|^{2} \leq\left(1+4 C_{\mathrm{PL}}\right)\left\|D^{s} u\right\|^{2}$ so that we can take $C_{\mathrm{K}} \leq 1+4 C_{\mathrm{PL}}$. This proves (8) since $D^{s} \mathcal{R}=\{0\}$.

$\triangleright$ Proof of (9). Let us take $u \in H^{1}$ such that $\langle u\rangle=0$ and $\mathbb{P}(u)=0$. By definition of $\mathbb{P}$, we have $\|u\|^{2} \leq\|u-\mathfrak{P}(D u) x\|$ since $x \mapsto \mathfrak{P}(D u) x$ is in $\mathcal{R}$. Applying (H3) and (8) gives

$$
\|u\|^{2} \leq C_{\mathrm{P}}\|D u-\mathfrak{P}(D u)\|^{2} \leq C_{\mathrm{P}} C_{\mathrm{K}}\left\|D^{s} u\right\|^{2}
$$

This proves (9) with $C_{\mathrm{PK}} \leq C_{\mathrm{P}} C_{\mathrm{K}}$.

$\triangleright$ Proof of (10). Let us take $u \in H^{1}$ such that $\langle u\rangle=0$ and $\mathbb{P}(u)=0$. Applying the strong Poincaré inequality (21) and the Korn inequality (8) gives

$$
\|\lfloor\nabla \phi\rceil u\|^{2} \leq C_{\mathrm{SP}}\|D u\|^{2}=C_{\mathrm{SP}}\left(\|D u-\mathfrak{P}(D u)\|^{2}+\|\mathfrak{P}(D u)\|^{2}\right) \leq C_{\mathrm{SP}} C_{\mathrm{K}}\left\|D^{s} u\right\|^{2}+C_{\mathrm{SP}}\|\mathfrak{P}(D u)\|^{2} .
$$

An integration by parts, Jensen's inequality and the Cauchy-Schwarz inequality show that

$$
\|\mathfrak{P}(D u)\|^{2}=\left|\left\langle D^{a} u\right\rangle\right|^{2}=\frac{1}{4} \sum_{i, j=1}^{d}\left|\int_{\mathbb{R}^{d}}\left(\partial_{j} \phi u_{i}-\partial_{i} \phi u_{j}\right) e^{-\phi} \mathrm{d} x\right|^{2} \leq\|\nabla \phi\|^{2}\|u\|^{2} .
$$

An integration by parts, $\Delta \phi \leq \sqrt{d}\left|D^{2} \phi\right|$ and (6) provide us with

$$
\int_{\mathbb{R}^{d}}|\nabla \phi|^{2} e^{-\phi} \mathrm{d} x=\int_{\mathbb{R}^{d}} \Delta \phi e^{-\phi} \mathrm{d} x \leq \frac{1}{4} \int_{\mathbb{R}^{d}}|\nabla \phi|^{2} e^{-\phi} \mathrm{d} x+\frac{1}{4}\left(C_{\phi}-1\right)
$$

so that $\|\nabla \phi\|^{2} \leq 3 C_{\phi}$ and we conclude that $\|\mathfrak{P}(D u)\|^{2} \leq 3 C_{\phi}\|u\|^{2} \leq 3 C_{\phi} C_{\mathrm{PK}}\left\|D^{s} u\right\|^{2}$ by (9). Inserting this estimate in (44) completes the proof of (10) with $C_{\mathrm{SPK}} \leq C_{\mathrm{SP}}\left(C_{\mathrm{K}}+3 C_{\phi} C_{\mathrm{PK}}\right)$.

\subsection{Proof of Theorem 2.}

$\triangleright$ Proof of (13). Since for any $R \in \mathcal{R}_{\phi}, D^{s} R=0$ and $\nabla \phi \cdot R=0$, we can consider $u \in H^{1}$ such that $\mathbb{P}_{\phi}(u)=0$ without loss of generality, so that $\mathbb{P}(u) \in \mathcal{R}_{\phi}^{c}$. According to (9) and by definition of the rigidity constant $C_{\mathrm{RV}}$ in (11), we have

$$
\begin{aligned}
\|u\|^{2}=\|u-\mathbb{P}(u)-\langle u\rangle\|^{2}+\| \mathbb{P}(u) & +\langle u\rangle\left\|^{2} \leq C_{\mathrm{PK}}\right\| D^{s} u\left\|^{2}+C_{\mathrm{RV}}\right\| \nabla \phi \cdot(\mathbb{P}(u)+\langle u\rangle) \|^{2} \\
& \leq C_{\mathrm{PK}}\left\|D^{s} u\right\|^{2}+2 C_{\mathrm{RV}}\|\nabla \phi \cdot u\|^{2}+2 C_{\mathrm{RV}}\|\nabla \phi \cdot(u-\mathbb{P}(u)-\langle u\rangle)\|^{2} .
\end{aligned}
$$

Applying then the strong Poincaré-Korn inequality (10) gives

$$
\|u\|^{2} \leq C_{\mathrm{PK}}\left\|D^{s} u\right\|^{2}+2 C_{\mathrm{RV}}\|\nabla \phi \cdot u\|^{2}+2 C_{\mathrm{RV}} C_{\mathrm{SPK}}\left\|D^{s} u\right\|^{2} .
$$

This completes the proof of (13) with $C_{\mathrm{PK}}^{\prime} \leq C_{\mathrm{PK}}+2 C_{\mathrm{RV}} C_{\mathrm{SPK}}$.

$\triangleright$ Proof of (14). Since for any $R \in \mathcal{R}_{\phi}, D^{s} R=0$ and $\nabla \phi \cdot R=0$, we can again consider $u \in H^{1}$ such that $\mathfrak{P}_{\phi}(D u)=0$ without loss of generality, so that $\mathfrak{P}(D u) \in \mathfrak{M}_{\phi}^{c}$. According to (8) and by definition of the rigidity constant $C_{\mathrm{RD}}$ in (12), we have

$$
\begin{aligned}
\|D u\|^{2}=\|D u-\mathfrak{P}(D u)\|^{2}+\|\mathfrak{P}(D u)\|^{2} \leq C_{\mathrm{K}}\left\|D^{s} u\right\|^{2}+C_{\mathrm{RD}}\|\nabla \phi \cdot(\mathfrak{P}(D u) x+\langle u\rangle)\|^{2} \\
\quad \leq C_{\mathrm{K}}\left\|D^{s} u\right\|^{2}+2 C_{\mathrm{RD}}\|\nabla \phi \cdot u\|^{2}+2 C_{\mathrm{RD}}\|\nabla \phi \cdot(u-\mathfrak{P}(D u) x-\langle u\rangle)\|^{2} .
\end{aligned}
$$

Applying the strong Poincaré inequality (21) gives

$$
\|D u\|^{2} \leq C_{\mathrm{K}}\left\|D^{s} u\right\|^{2}+2 C_{\mathrm{RD}}\|\nabla \phi \cdot u\|^{2}+2 C_{\mathrm{RD}} C_{\mathrm{SP}}\|D u-\mathfrak{P}(D u)\|^{2},
$$

and by the Korn inequality (8) again,

$$
\|D u\|^{2} \leq C_{\mathrm{K}}\left\|D^{s} u\right\|^{2}+2 C_{\mathrm{RD}}\|\nabla \phi \cdot u\|^{2}+2 C_{\mathrm{RD}} C_{\mathrm{SP}} C_{\mathrm{K}}\left\|D^{s} u\right\|^{2},
$$

This gives (13) with $C_{\mathrm{K}}^{\prime} \leq C_{\mathrm{K}}\left(1+2 C_{\mathrm{RD}} C_{\mathrm{SP}}\right)$, with $C_{\mathrm{SP}} \leq C_{\phi}\left(1+C_{\mathrm{P}}\right)$ according to Proposition 5 . 


\section{Operators on VeCtor Fields: Proof of TheOrem 3}

In this section we develop the functional analysis and the spectral theory of operators on vector fields, and prove Theorem 3. All results on the tensorized operator $-\Delta_{\phi}$ on vector fields are direct consequences of the study of the corresponding scalar operator: from Section 3 , we learn that $-\Delta_{\phi}$ is essentially self-adjoint and admits $\mathcal{C}_{c}^{\infty}\left(\mathbb{R}^{d} ; \mathbb{R}^{d}\right)$ as a core, the domain of its unique self-adjoint extension is

$$
D\left(-\Delta_{\phi}\right)=\left\{u \in L^{2}: \forall j \in\{1, \cdots, d\},\left\|\lfloor\nabla \phi\rceil^{2} u_{j}\right\|^{2}+\left\|\lfloor\nabla \phi\rceil \nabla u_{j}\right\|^{2}+\left\|D^{2} u_{j}\right\|^{2}<\infty\right\},
$$

and its kernel is $\operatorname{ker}\left(-\Delta_{\phi}\right)=\mathbb{R}^{d}$.

Let us deal with the other operators of Theorem 3 . Recall that the operator $-\Delta_{S}$ is defined on $\mathcal{C}_{c}^{\infty}\left(\mathbb{R}^{d} ; \mathbb{R}^{d}\right)$ vector fields by $-\Delta_{S}=-D_{\phi}^{s} \cdot D^{s}$. It is nonnegative and Id $-\Delta_{S}$ has therefore a Friedrichs extension with domain included in $H_{S}^{1}$ defined as the completion of $\mathcal{C}_{c}^{\infty}\left(\mathbb{R}^{d} ; \mathbb{R}^{d}\right)$ with respect to the norm given by $u \mapsto\|u\|^{2}+\left\|D^{s} u\right\|^{2}$. On the other hand, a maximal self-adjoint extension of $\mathrm{Id}-\Delta_{S}$ can be built according to the Lax-Milgram Theorem and its domain is included in $H^{1}$. The Korn inequality (8) implies that $H_{S}^{1}=H^{1}$ so that the two extensions coincide, since there is a unique extension for which the domain is contained in $H_{S}^{1}\left(\left[37\right.\right.$, Theorem X.23]), which is the case for the maximal one. We have proven that $-\Delta_{S}$ is essentially self-adjoint. From the Poincaré-Korn inequality (9), we learn that $\operatorname{ker}\left(-\Delta_{S}\right)=\mathcal{R} \oplus \mathbb{R}^{d}$ and that $\inf \left(\operatorname{Sp}\left(-\Delta_{S}\right) \cap(0,+\infty)\right) \geq C_{\mathrm{PK}}^{-1}>0$. This concludes the proof of Theorem 3 for $-\Delta_{S}$.

The same argument applies to $-\Delta_{S \phi}=-\Delta_{S}-\nabla \phi \otimes \nabla \phi$ using (8)-(10) as we know from Proposition 8 that $\nabla \phi \otimes \nabla \phi u \in L^{2}$ for all $u \in D$. This completes the proof of of Theorem 3 .

Remark 9. Note also that the alternative operator defined on smooth vector fields by $u \mapsto-D_{\phi}^{s} \cdot D^{s} u-$ $\nabla\left(\nabla_{\phi} \cdot u\right)$ has exactly the same properties as $-\Delta_{S}$ because $\left\|D^{s} u\right\|^{2}+\|\nabla \phi \cdot u\|^{2} \sim\left\|D^{s} u\right\|^{2}+\left\|\nabla_{\phi} \cdot u\right\|^{2}$ where $\nabla_{\phi} u:=\nabla \cdot u-\nabla \phi \cdot u$ and $\nabla \cdot u=\operatorname{Tr}\left(D^{s} u\right)$.

\section{Zeroth order Korn inequalities: Proof of TheOrem 4}

In order to prove (18), we use a new Poincaré-Lions-type inequality of order -1 and the Schwarz Lemma.

\subsection{A Poincaré-Lions inequality of order -1 .}

Lemma 10. There exists two positive constants $C_{\mathrm{LPL}}$ and $C_{\mathrm{RPL}}$ such that, for all $f \in H^{-1}$, we have

$$
C_{\mathrm{LPL}}^{-1}\left\|\Lambda^{-1 / 2}(f-\langle f\rangle)\right\|^{2} \leq\left\|\Lambda^{-1} \nabla f\right\|^{2} \leq C_{\mathrm{RPL}}\left\|\Lambda^{-1 / 2}(f-\langle f\rangle)\right\|^{2} .
$$

Proof. We rely on the same strategy as for the proof of the Poincaré-Lions inequality (22). For any $f \in H^{-1}$, the mean makes sense because $\langle f\rangle=\Lambda^{-1 / 2}\langle f\rangle=\left\langle\Lambda^{-1 / 2} f\right\rangle$ as $\Lambda=$ Id when restricted on constants. We can therefore take $\langle f\rangle=0$ w.l.o.g. and apply the spectral theorem as in (39), for any $f \in D(\Lambda)$, to get

$$
\left(1+C_{\mathrm{P}}\right)^{-1}\left\|\Lambda^{-1 / 2} f\right\|^{2} \leq\left(\left(-\Delta_{\phi}\right) \Lambda^{-1} \Lambda^{-1 / 2} f, \Lambda^{-1 / 2} f\right)=\left(\Lambda \nabla \Lambda^{-3 / 2}\left(\Lambda^{-1 / 2} f\right), \Lambda^{-1} \nabla f\right),
$$

where we used that $-\Delta_{\phi}=-\nabla_{\phi} \cdot \nabla$ and $\Lambda$ commute. In order to prove the left inequality in (46), it is sufficient to prove that $\Lambda \nabla \Lambda^{-3 / 2}$ is a bounded operator. We work in $\mathcal{C}_{c}^{\infty}\left(\mathbb{R}^{d} ; \mathbb{R}\right)$, which is a core for $\Lambda$, and the conclusion follows by density in $L^{2}$. Let us write

$$
\Lambda \nabla \Lambda^{-3 / 2}=\nabla \Lambda^{-1 / 2}+[\Lambda, \nabla] \Lambda^{-3 / 2}=\nabla \Lambda^{-1 / 2}-D^{2} \phi \nabla \Lambda^{-3 / 2}=\nabla \Lambda^{-1 / 2}-D^{2} \phi \Lambda^{-1}\left(\Lambda \nabla \Lambda^{-3 / 2}\right) .
$$

By assumption $(\mathrm{H} 2)$, for all $\varepsilon>0$ and for all $g \in \mathcal{C}^{\infty}\left(\mathbb{R}^{d} ; \mathbb{R}\right)$, we know that

$$
\begin{aligned}
\left\|\Lambda \nabla \Lambda^{-3 / 2} g\right\| & \leq\left\|\nabla \Lambda^{-1 / 2} g\right\|+\varepsilon\left\|\lfloor\nabla \phi\rceil^{2} \nabla \Lambda^{-3 / 2} g\right\|+C_{\varepsilon}\left\|\nabla \Lambda^{-3 / 2} g\right\| \\
& \left.\leq\left\|\nabla \Lambda^{-1 / 2} g\right\|+\varepsilon \| \mid \nabla \phi\right\rceil^{2} \Lambda^{-1}\left(\Lambda \nabla \Lambda^{-3 / 2} g\right)\left\|+C_{\varepsilon}\right\| \nabla \Lambda^{-1 / 2}\left(\Lambda^{-1} g\right) \| .
\end{aligned}
$$

The operators $\nabla \Lambda^{-1 / 2}$ and $\lfloor\nabla \phi\rceil^{2} \Lambda^{-1}$ are bounded respectively by 1 and $\sqrt{C_{\mathrm{B}}}$ according to (28) and (31), and $\Lambda^{-1} \leq 1$, so that

$$
\left\|\Lambda \nabla \Lambda^{-3 / 2} g\right\| \leq\left(1+C_{\varepsilon}\right)\|g\|+\varepsilon \sqrt{C_{\mathrm{B}}}\left\|\Lambda \nabla \Lambda^{-3 / 2} g\right\|
$$

With the choice $\varepsilon=1 /\left(2 \sqrt{C_{\mathrm{B}}}\right)$ and $C_{\phi}^{\prime \prime}:=C_{\varepsilon}=C_{1 /\left(2 \sqrt{C_{\mathrm{B}}}\right)}$ in (H2), we obtain

$$
\left\|\Lambda \nabla \Lambda^{-3 / 2} g\right\| \leq 2\left(1+C_{\phi}^{\prime \prime}\right)\|g\| .
$$

Coming back to (47) with $g=\Lambda^{-1 / 2} f$, we obtain

$$
\left\|\Lambda^{-1 / 2} f\right\| \leq 2\left(1+C_{\mathrm{P}}\right)\left(1+C_{\phi}^{\prime \prime}\right)\left\|\Lambda^{-1} \nabla f\right\|,
$$

so that $C_{\mathrm{LPL}} \leq 4\left(1+C_{\mathrm{P}}\right)^{2}\left(1+C_{\phi}^{\prime \prime}\right)^{2}$ and the left inequality is proven. 
In order to prove the right inequality in (46), we notice that $\Lambda^{-1} \nabla f=\Lambda^{-1} \nabla \Lambda^{1 / 2}\left(\Lambda^{-1 / 2} f\right)$ and it is therefore sufficient to prove that $\Lambda^{-1} \nabla \Lambda^{1 / 2}$ is a bounded operator. This is done as in (40) by writing

$$
\Lambda^{-1} \nabla \Lambda^{1 / 2}=\Lambda^{-1} \nabla \Lambda \Lambda^{-1 / 2}=\nabla \Lambda^{-1 / 2}+\Lambda^{-1}[\nabla, \Lambda] \Lambda^{-1 / 2}=\nabla \Lambda^{-1 / 2}+\Lambda^{-1} D^{2} \phi\left(\nabla \Lambda^{-1 / 2}\right) .
$$

As in the proof of $(40)$, we obtain for any $f \in \mathcal{C}_{c}^{\infty}\left(\mathbb{R}^{d} ; \mathbb{R}\right)$ and $g=\Lambda^{-1 / 2} f$ the estimate

$$
\left\|\Lambda^{-1} \nabla f\right\|=\left\|\Lambda^{-1} \nabla \Lambda^{1 / 2} g\right\| \leq\left(1+\frac{1}{4} C_{\phi}^{\prime} \sqrt{C_{\mathrm{B}} / C_{\phi}}\right)\left\|\nabla \Lambda^{-1 / 2} g\right\| \leq\left(1+\frac{1}{4} C_{\phi}^{\prime} \sqrt{C_{\mathrm{B}} / C_{\phi}}\right)\|g\|,
$$

using (6), (28) and (32). This concludes the proof with $C_{\mathrm{RPL}}=\left(1+\frac{1}{4} C_{\phi}^{\prime} \sqrt{C_{\mathrm{B}} / C_{\phi}}\right)^{2}$.

6.2. Proof of the Korn inequalities in Theorem 4. As a consequence of (45) the projection $u \mapsto$ $\mathfrak{P}(D u)=\left\langle D^{a} u\right\rangle$ has a unique extension as a bounded operator on $L^{2}$ with norm bounded by $\|\nabla \phi\|$ since $H^{1}$ is dense in $L^{2}$. We keep the same name for the extension and notice that

$$
\Lambda^{-1 / 2} \mathfrak{P}(D u)=\Lambda^{-1 / 2}\left\langle D^{a} u\right\rangle=\left\langle\Lambda^{-1 / 2} D^{a} u\right\rangle=\mathfrak{P}\left(\Lambda^{-1 / 2} D u\right) .
$$

$\triangleright$ Proof of (18). Let us take $u \in L^{2}$ such that $\langle u\rangle=0$ and $\mathfrak{P}(D u)=\left\langle D^{a} u\right\rangle=0$. Using (46), we have

$$
\left\|\Lambda^{-1 / 2} D u\right\|^{2}=\left\|\Lambda^{-1 / 2} D^{s} u\right\|^{2}+\left\|\Lambda^{-1 / 2} D^{a} u\right\|^{2} \leq\left\|\Lambda^{-1 / 2} D^{s} u\right\|^{2}+C_{\mathrm{LPL}}\left\|\Lambda^{-1} \nabla\left(D^{a} u\right)\right\|^{2}
$$

where $\left\|\Lambda^{-1} \nabla\left(D^{a} u\right)\right\|^{2}=\sum_{i, j=1}^{d}\left\|\Lambda^{-1} \nabla\left(D^{a} u\right)_{i j}\right\|^{2}$. By the Schwarz Theorem (23),

$$
\left\|\Lambda^{-1} \nabla\left(D^{a} u\right)\right\|^{2} \leq 2 \sum_{i, j, k=1}^{d}\left(\left\|\Lambda^{-1} \partial_{i}\left(D^{s} u\right)_{j k}\right\|^{2}+\left\|\Lambda^{-1} \partial_{j}\left(D^{s} u\right)_{i k}\right\|^{2}\right)=4 \sum_{j, k=1}^{d}\left\|\Lambda^{-1} \nabla\left(D^{s} u\right)_{j k}\right\|^{2},
$$

and (46) yields

$$
\sum_{i, j=1}^{d}\left\|\Lambda^{-1} \nabla\left(D^{s} u\right)_{i j}\right\|^{2} \leq C_{\mathrm{RPL}} \sum_{j, k=1}^{d}\left\|\Lambda^{-1 / 2}\left(D^{s} u\right)_{j k}\right\|^{2}=C_{\mathrm{RPL}}\left\|\Lambda^{-1 / 2} D^{s} u\right\|^{2} .
$$

Altogether, this proves

$$
\left\|\Lambda^{-1 / 2}(D u-\mathfrak{P}(D u))\right\|^{2} \leq\left(1+4 C_{\mathrm{LPL}} C_{\mathrm{RPL}}\right)\left\|\Lambda^{-1 / 2} D^{s} u\right\|^{2}
$$

and (18) follows with $C_{\mathrm{K} 0}=1+4 C_{\mathrm{LPL}} C_{\mathrm{RPL}}$.

$\triangleright$ Proof of (19). Let us take $u \in L^{2}$ such that $\langle u\rangle=0$ and $\mathbb{P}(u)=0$. By definition of $\mathbb{P},(22)$ and (18) we get

$$
\|u\|^{2} \leq\|u-\mathfrak{P}(D u) x\| \leq C_{\mathrm{PL}}\left\|\Lambda^{-1 / 2}(D u-\mathfrak{P}(D u))\right\|^{2} \leq C_{\mathrm{PL}}\left(1+4 C_{\mathrm{LPL}} C_{\mathrm{RPL}}\right)\left\|\Lambda^{-1 / 2} D^{s} u\right\|^{2} .
$$

This proves (19) with $C_{\mathrm{PK} 0} \leq C_{\mathrm{PL}}\left(1+4 C_{\mathrm{LPL}} C_{\mathrm{RPL}}\right)$.

$\triangleright$ Proof of (20). Let us consider $u \in L^{2}$ such that $\mathbb{P}_{\phi}(u)=0$ so that $\mathbb{P}(u) \in \mathcal{R}_{\phi}^{c}$. By (19) and by definition (17), we have

$$
\begin{aligned}
\|u\|^{2} & =\|u-\mathbb{P}(u)-\langle u\rangle\|^{2}+\|\mathbb{P}(u)+\langle u\rangle\|^{2} \\
& \leq C_{\mathrm{PKo}}\left\|\Lambda^{-1 / 2} D^{s} u\right\|^{2}+C_{\mathrm{RVO}}\left\|\Lambda^{-1 / 2} \nabla \phi \cdot(\mathbb{P}(u)+\langle u\rangle)\right\|^{2} \\
& \leq C_{\mathrm{PKo}}\left\|\Lambda^{-1 / 2} D^{s} u\right\|^{2}+2 C_{\mathrm{RVo}}\left\|\Lambda^{-1 / 2}(\nabla \phi \cdot u)\right\|^{2}+2 C_{\mathrm{RVo}}\left\|\Lambda^{-1 / 2}[\nabla \phi \cdot(u-\mathbb{P}(u)-\langle u\rangle)]\right\|^{2} .
\end{aligned}
$$

Inequalities (29), $\Lambda^{-1 / 2} \leq 1$ and (19) yield

$$
\begin{aligned}
\|u\|^{2} & \leq C_{\mathrm{PKo}}\left\|\Lambda^{-1 / 2} D^{s} u\right\|^{2}+2 C_{\mathrm{RV0}}\left\|\Lambda^{-1 / 2}(\nabla \phi \cdot u)\right\|^{2}+2 C_{\mathrm{RV} 0} C_{\phi}\|u-\mathbb{P}(u)-\langle u\rangle\|^{2} \\
& \leq C_{\mathrm{PKo}}\left\|\Lambda^{-1 / 2} D^{s} u\right\|^{2}+2 C_{\mathrm{RVo}}\left\|\Lambda^{-1 / 2}(\nabla \phi \cdot u)\right\|^{2}+2 C_{\mathrm{RV} 0} C_{\phi} C_{\mathrm{PKo}}\left\|\Lambda^{-1 / 2} D^{s} u\right\|^{2} .
\end{aligned}
$$

This proves (20) with $C_{\mathrm{PK} 0}^{\prime} \leq C_{\mathrm{PK} 0}\left(1+2 C_{\mathrm{RV} 0} C_{\phi}\right)$, and also completes the proof of Theorem 4 . 


\section{Appendix A. Extensions, GEOMETRIC OBSERVATIONs AND MOTIVATiOn From KinetiC THEORY}

\section{A.1. On the assumptions and some generalizations.}

Remark 11. The fact that the Poincaré inequality (H3) implies that $e^{-\phi} \mathrm{d} x$ has an average $\langle x\rangle$ and a variance $\left\langle|x|^{2}\right\rangle$ is classical (see, e.g., [31, Corollary 3.2]). Indeed (H3) yields a concentration property of $e^{-\phi(x)} \mathrm{d} x$ via a concentration function. A direct application of Fatou's Lemma allows to extend (H3) to the set $W^{1, \infty}$ of uniformly Lipschitz functions, which includes $x \mapsto x_{j}$ for all $j \in\{1, \cdots, d\}$. This directly gives $\int_{\mathbb{R}^{d}}|x|^{2} e^{-\phi(x)} \mathrm{d} x \leq d C_{\mathrm{P}}$ and the integrability of $x$ w.r.t. $e^{-\phi(x)} \mathrm{d} x$ follows by the Cauchy-Schwarz inequality. By induction, we get under (H3) alone that the set of all polynomial functions $\mathbb{R}[x]$ is included in $L^{2}$ and even $H^{1}$.

Remark 12. As a consequence of Remark 11 and of the strong Poincaré inequality (21), we directly get that for all $i, j \in\{1, \cdots d\}, \int_{\mathbb{R}^{d}}\left|x_{i} \partial_{j} \phi(x)\right| e^{-\phi(x)} \mathrm{d} x<\infty$. It is indeed sufficient to apply the strong Poincaré inequality to $x \mapsto x_{j}$, which is in $H^{1}$. Note that this gives sense to all quantities of Theorem 1, e.g., $\|\nabla \phi \cdot R\|$ for any infinitesimal rotation $R$.

Remark 13. There are many sufficient conditions for the Poincaré inequality of Assumption (H3). When $\phi$ is uniformly convex, it is shown in [3] that $C_{\mathrm{P}}$ is greater or equal than the convexity constant, hence leading to fully explicit estimates in the two main theorems. If we only assume that $\lim _{|x| \mapsto \infty}|\nabla \phi(x)|=+\infty$, then $\Lambda$ is in fact an operator with compact resolvent, hence with discrete spectrum, and (H3) follows. Another, less stringent, sufficient condition on $\phi$ is $\liminf _{|x| \rightarrow \infty}\left(\frac{1}{2}|\nabla \phi(x)|^{2}-\Delta \phi(x)\right)>c$ for some $c>0$ (it implies the Poincaré inequality from the Persson-Agmon formula of [36] or [1, Theorem 3.2]). We note that this last assumption is satisfied by any regular function which coincides with $x \mapsto \alpha|x|+\beta$ outside of a large centred ball, where $\alpha$ and $\beta$ are normalization constants.

Remark 14. Assumptions (H1)-(H2)-(H3) may be satisfied in other geometries than the one of the whole Euclidean space $\mathbb{R}^{d}$. In particular, given an open, smooth, bounded and connected subset $\Omega$ of $\mathbb{R}^{d}$, we observe that these hypotheses are satisfied by the potential $\phi(x)=\exp \left(1 / d^{2}(x, \partial \Omega)\right)$, where $d(x, \partial \Omega)$ denotes the usual Euclidean distance from $x$ to $\partial \Omega$. Here $\mathbb{R}^{d}$ is replaced by $\Omega$ equipped with the measure $e^{-\phi(x)} \mathrm{d} x$. It is an open question to understand how our results could be extended to usual boundary problems with potentials mimicking walls at the boundary of $\Omega$.

A.2. Rigidity constants and defects of axisymmetry. In the proofs of Theorems 1 and 4 (see also Appendix B.5), we used the two rigidity constants $C_{\mathrm{RV}}$ and $C_{\mathrm{RV} 0}$ defined by (11) and (17), depending on the level of regularity in each case, to measure the defects of axisymmetry (note that $C_{\mathrm{RV}} \leq C_{\mathrm{Rvo}}$ since $\Lambda \geq \mathrm{Id}$ ). We used also $C_{\mathrm{RD}}$ defined in (12) but remark that $C_{\mathrm{RV}}$ and $C_{\mathrm{RD}}$ are not directly comparable either, because $\mathfrak{M}_{\phi}^{c} \neq D \mathcal{R}_{\phi}^{c}$. Other ways of measuring the default of axisymmetry of the potential $\phi$ can be considered.

(1) One can consider, again, a rigidity of vector fields constant, but this time defined alternatively by

$$
C_{\mathrm{RVL}}^{-1}=\min _{A x \in \mathcal{R}_{\phi}^{c} \backslash\{0\}} \frac{\|\nabla \phi \cdot A x\|^{2}}{\|A x\|^{2}} \text { when } \quad \mathcal{R}_{\phi}^{c} \neq\{0\} \quad \text { and } \quad C_{\mathrm{RVL}}=0 \quad \text { otherwise. }
$$

This leads to the modified Poincaré-Korn inequality

$$
\inf _{A x \in \mathcal{R}_{\phi}}\|u-\langle u\rangle-A x\|^{2}=\left\|u-\langle u\rangle-\mathbb{P}_{\phi}(u)\right\|^{2} \leq C_{\mathrm{PKL}}^{\prime}\left\|D^{s} u\right\|^{2}+2 C_{\mathrm{RVL}}\|\nabla \phi \cdot(u-\langle u\rangle)\|^{2}
$$

with an explicit bound for the constant $C_{\mathrm{PKL}}^{\prime}$ using (9) and the method of proof of (13). Once more, the existence of $C_{\mathrm{RVL}}$ follows from the injectivity of $A x \mapsto \nabla \phi \cdot A x$ on $\mathcal{R}_{\phi}^{c}$ and the fact that $\mathcal{R}_{\phi}^{c}$ is of finite dimension. The main advantage of this approach is to preserve a continuity property with respect to axisymmetry, which can be stated as follows: a small perturbation of a radial potential $\phi$ gives rise to a small constant $C_{\mathrm{RVL}}$, the limiting case being $\mathcal{R}_{\phi}^{c}=\{0\}$ and $C_{\mathrm{RVL}}=0$. The main drawback is that the symmetric operator associated to (48) is neither local nor differential because of the term $\langle u\rangle$ which appears in the right-hand side of (48).

(2) In a bounded domain $\Omega \subset \mathbb{R}^{d}$, with flat metric (i.e., for a constant potential $\phi$ ) considered in [12], the authors use Grad's number. Let us explain how to adapt this method in our context under, e.g., the additional condition

$$
\lim _{|x| \rightarrow \infty} \frac{D^{2} \phi(x)}{[\nabla \phi(x)\rceil^{2}}=0
$$


This property implies that the multiplication operator by $D^{2} \phi$ is relatively compact with respect to $-\Delta_{\phi}$ acting on vector fields, with essential spectrum in $\left[C_{\mathrm{P}}, \infty\right)$. The spectrum in $\left[0, C_{\mathrm{P}}\right)$ is then a pure point spectrum and the kernel is finite dimensional. For any antisymmetric matrix $A$, there exists an affine space $\mathfrak{V}_{A}$ of functions $v \in H^{1}$ solving the Witten-Hodge problem

$$
\operatorname{div}_{\phi} v=0, \quad D^{a} v=A .
$$

The Witten-Hodge inequality asserts that

$$
\inf _{v \in \mathfrak{V}_{A}}\left\|D^{s} v\right\|^{2} \leq c_{\mathrm{H}}|A|^{2}
$$

for some constant $c_{\mathrm{H}} \in(0, \infty)$. The reverse inequality amounts to the existence of Grad's number such that

$$
C_{\mathrm{G}}^{-1}:=\inf _{A \in \mathfrak{M}_{\phi}^{c},|A|=1, v \in \mathfrak{V}_{A}}\left\|D^{s} v\right\|^{2} .
$$

The existence of $C_{\mathrm{G}}$ as well as a quantitative positive lower bound could be establish using mass transport theory exactly as in [12]. Of course, $C_{\mathrm{G}}$ is well defined only when $\mathcal{R}_{\phi}^{c} \neq\{0\}$, i.e., under the condition that $\phi$ is not radially symmetric. Inequality (49) is natural in differential geometry and more specifically in De Rham cohomology theory: we refer to $[2,21]$ for further developments on this topic. In bounded domains, how to measure the symmetry defect by Grad's number in view of Korn type inequalities is at the core of [12] but has also been studied in [18]. This approach differs from ours. Using $C_{\mathrm{G}}$ and (8), the inequality (13) can be proved along a similar strategy as in [12], although with different constants.

A.3. An elementary application in kinetic theory. The main motivation for this paper comes from kinetic equations involving a confining potential studied in [7]. Also see [12, Section 2] and [4, 15] for applications of Korn inequalities to kinetic equations. As an example, let us consider the linear relaxation model of BGK-type

$$
\partial_{t} f+v \cdot \nabla_{x} f-\nabla_{x} \phi \cdot \nabla_{v} f=\mathcal{L} f:=G_{f}-f,
$$

where $f(t, x, v)$ is an unknown distribution function for a system of particles depending on time $t \geq 0$, position $x \in \mathbb{R}^{d}$ and velocity $v \in \mathbb{R}^{d}$, and where $G_{f}$ is defined by

$$
G_{f}:=(\rho+\mathbf{u} \cdot v) \mu \quad \text { where } \quad \rho(t, x):=\int_{\mathbb{R}^{d}} f(t, x, v) \mathrm{d} v, \quad \mathbf{u}(t, x):=\int_{\mathbb{R}^{d}} v f(t, x, v) \mathrm{d} v .
$$

Here $\mu(v):=(2 \pi)^{-d / 2} e^{-|v|^{2} / 2}$ while $\rho$ and $\mathbf{u}$ are respectively the macroscopic density and the average velocity associated with $f$. The collision kernel admits $d+1$ conserved moments, in the sense that $\int_{\mathbb{R}^{d}} \mathcal{L} f(t, x, v) \mathrm{d} v=0=\int_{\mathbb{R}^{d}} v_{i} \mathcal{L} f(t, x, v) \mathrm{d} v$ for any $i=1, \ldots, d$ and $f \in L^{1}((1+|v|) \mathrm{d} v)$.

A natural question is to look for equilibria of (50). A quick glance at the equation shows that $\mathcal{M}(x, v):=$ $e^{-\phi(x)} \mu(v)$ is one of them. Korn inequalities provide us with a complete answer.

Proposition 15. Under Assumptions (H1)-(H2)-(H3), all equilibria of (50) in $L^{2}\left(\mathcal{M}^{-1} \mathrm{~d} x \mathrm{~d} v\right)$ take the form $f(x, v)=((R(x) \cdot v)+c) \mathcal{M}$ for some $R \in \mathcal{R}_{\phi}$ and $c \in \mathbb{R}$.

Proof. Write $f=h \mathcal{M}$ with $h \in L^{2}(\mathcal{M} \mathrm{d} x \mathrm{~d} v), \rho=r e^{-\phi}, \mathbf{u}=u e^{-\phi}$, so that equation (50) reads

$$
\partial_{t} h+v \cdot \nabla_{x} h-\nabla_{x} \phi \cdot \nabla_{v} h=L(h):=h-r-u \cdot v .
$$

The restriction of $L$ to $L^{2}(\mu \mathrm{d} v)$ is $L=-\Pi^{\perp}$ where $\Pi$ is the orthogonal projection onto $\operatorname{Span}\left\{1, v_{1}, \ldots, v_{d}\right\}$. We compute

$$
\frac{d}{d t} \iint_{\mathbb{R}^{d} \times \mathbb{R}^{d}}|h|^{2} \mathcal{M} \mathrm{d} x \mathrm{~d} v=2 \iint_{\mathbb{R}^{d} \times \mathbb{R}^{d}}(L h) h \mathcal{M} \mathrm{d} x \mathrm{~d} v=-2 \iint_{\mathbb{R}^{d} \times \mathbb{R}^{d}}\left|\Pi^{\perp} h\right|^{2} \mathcal{M} \mathrm{d} x \mathrm{~d} v
$$

and deduce that any stationary solution of (51) takes the form $h(x, v)=r(x)+u(x) \cdot v$. Equation (51) then reads

$$
v \cdot \nabla_{x}(r+u \cdot v)=\nabla_{x} \phi \cdot u .
$$

Integrating the latter equation against respectively $1, v_{i}$ and $v_{i} v_{j}$ with $i \neq j$ in $L^{2}(\mu \mathrm{d} v)$ yields

$$
\text { i) } \nabla_{x} \cdot u-\nabla_{x} \phi \cdot u=0, \quad \text { ii) } \quad D^{s} u=0, \text { iii) } \quad \nabla r=0 .
$$

From iii) we get that there exists $c \in \mathbb{R}$ such that $r=c$. As for i), an integration by parts gives

$0=\int_{\mathbb{R}^{d}}\left(\nabla_{x} \cdot u-\nabla_{x} \phi \cdot u\right)\langle u\rangle \cdot x e^{-\phi(x)} \mathrm{d} x=-\int_{\mathbb{R}^{d}} u \cdot \nabla(\langle u\rangle \cdot x) e^{-\phi(x)} \mathrm{d} x=-\int_{\mathbb{R}^{d}} u \cdot\langle u\rangle e^{-\phi(x)} \mathrm{d} x=-\langle u\rangle^{2}$, 
so that $\langle u\rangle=0$. Note also that taking the trace in ii) yields $\nabla_{x} \cdot u=0$ so that i) reads $\nabla_{x} \phi \cdot u=0$. Using this and (19) in Theorem 4 shows that $u=R$ with $R=\mathbb{P}_{\phi}(u) \in \mathcal{R}_{\phi}$. Hence $h=R(x) \cdot v+c$ and $f(x, v)=((R(x) \cdot v)+c) \mathcal{M}$. The reciprocal is straightforward, which completes the proof.

\section{Appendix B. Additional details on computations}

B.1. Functions, derivatives and projections. We denote by $f$ a generic scalar function on $\mathbb{R}^{d}$ and by $u: \mathbb{R}^{d} \rightarrow \mathbb{R}^{d}$ a generic vector field, so that $\nabla f=\left(\partial_{i} f\right)_{i=1}^{d}$ is a vector field and $D u=\left(\partial_{j} u_{i}\right)_{i, j=1}^{d}$ takes values in $\mathfrak{M}$. The symmetric and the antisymmetric differentials of $u$, respectively $D^{s} u=\left(\left(D^{s} u\right)_{i j}\right)_{i, j=1}^{d}$ and $D^{a} u=\left(\left(D^{a} u\right)_{i j}\right)_{i, j=1}^{d}$ are defined by

$$
\left(D^{s} u\right)_{i j}:=\frac{1}{2}\left(\partial_{j} u_{i}+\partial_{i} u_{j}\right) \quad \text { and } \quad\left(D^{a} u\right)_{i j}:=\frac{1}{2}\left(\partial_{j} u_{i}-\partial_{i} u_{j}\right)
$$

so that $D^{s} u+D^{a} u=D u$.

The orthogonal projection $\mathbb{P}$ of vector-valued functions is defined as follows. Let $\left(A_{i j}\right)_{1 \leq i<j \leq d}$ be a basis of $\mathfrak{M}^{a}$ whose elements are

$$
A_{i j}=\left(\left(\delta_{i j}-\delta_{j i}\right) \delta_{k i} \delta_{j \ell}\right)_{k, \ell=1}^{d}
$$

and $\left(R_{i j}\right)_{1 \leq i<j \leq d}$ the orthonormal (in the $L^{2}$ sense) basis of $\mathcal{R}$ given by

$$
R_{i j}(x)=Z_{i j}^{-1} A_{i j} x
$$

whose coordinates are all 0 except the $i^{t h}$ and the $j^{t h}$ ones, with respective values $-x_{j} / Z_{i j}$ and $x_{i} / Z_{i j}$, i.e.,

$$
R_{i j}(x)^{\perp}=Z_{i j}^{-1}\left(0, \ldots, 0,-x_{j}, 0, \ldots, 0, x_{i}, 0, \ldots, 0\right),
$$

and where the normalization constant is $Z_{i j}=\left(\int_{\mathbb{R}^{d}}\left(x_{i}^{2}+x_{j}^{2}\right) e^{-\phi(x)} \mathrm{d} x\right)^{1 / 2}$. With these notations, $\mathbb{P} u$ is the vector field

$$
x \mapsto \mathbb{P} u(x):=\sum_{1 \leq i<j \leq d} \mathrm{c}_{i j} R_{i j}(x)
$$

where the coefficients are computed, for all integers $i, j$ such that $1 \leq i<j \leq d$, as

$$
\mathrm{c}_{i j}=\int_{\mathbb{R}^{d}} u(x) \cdot R_{i j}(x) e^{-\phi(x)} \mathrm{d} x=\frac{1}{Z_{i j}} \int_{\mathbb{R}^{d}}\left(x_{i} u_{j}(x)-x_{j} u_{i}(x)\right) e^{-\phi(x)} \mathrm{d} x .
$$

The orthogonal projection $\mathfrak{P}$ of a matrix-valued function $\mathfrak{F}$ is defined as

$$
\mathfrak{P F}:=\sum_{1 \leq i<j \leq d} \mathrm{~d}_{i j} A_{i j}
$$

where the coefficients are computed, for all integers $i, j$ such that $1 \leq i<j \leq d$, as

$$
\mathrm{d}_{i j}=\frac{1}{2} \int_{\mathbb{R}^{d}} \mathfrak{F}(x): A_{i j} e^{-\phi(x)} \mathrm{d} x=\frac{1}{2} \int_{\mathbb{R}^{d}}\left(\mathfrak{F}_{i j}(x)-\mathfrak{F}_{j i}(x)\right) e^{-\phi(x)} \mathrm{d} x .
$$

As a consequence, we deduce that $\mathfrak{P F}=\left\langle\mathfrak{F}^{a}\right\rangle$ and $\mathfrak{P}(D u)=\left\langle D^{a} u\right\rangle$ for any $u \in H^{1}$.

A matrix $A \in D \mathcal{R}_{\phi}^{c}$ is such that $A \in \mathfrak{M}^{a}$ and for any $B \in D \mathcal{R}_{\phi} \subset \mathfrak{M}^{a}$,

$$
0=\int_{\mathbb{R}^{d}} A x \cdot B x e^{-\phi(x)} \mathrm{d} x=\sum_{i, j, k=1}^{d} A_{i j} B_{i k} \int_{\mathbb{R}^{d}} x_{j} x_{k} e^{-\phi(x)} \mathrm{d} x .
$$

A matrix $A \in \mathfrak{M}_{\phi}^{c}$ is such that $A \in \mathfrak{M}^{a}$ and for any $B \in D \mathcal{R}_{\phi}=\mathfrak{M}_{\phi} \subset \mathfrak{M}^{a}$,

$$
0=\int_{\mathbb{R}^{d}} A: B e^{-\phi(x)} \mathrm{d} x=A: B=\sum_{i, j}^{d} A_{i j} B_{i j} .
$$

Based on these two definitions, it is clear that $D \mathcal{R}_{\phi}^{c}$ and $\mathfrak{M}_{\phi}^{c}$ generically differ.

B.2. Operators. Let us give some details on the differential operators $-\Delta_{\phi}$ and $-\Delta_{S}$ associated respectively with the quadratic forms $f \mapsto\|\nabla f\|^{2}$ and $u \mapsto\left\|D^{s} u\right\|^{2}$.

$\triangleright$ Using $\nabla_{\phi} u:=\nabla \cdot u-\nabla \phi \cdot u$, the Witten-Laplace operator $\Delta_{\phi}$ on functions is such that $\|\nabla f\|^{2}=\left(f,-\Delta_{\phi} f\right)$ and takes the form

$$
\Delta_{\phi} f=e^{\phi} \nabla \cdot\left(\nabla f e^{-\phi}\right)=\nabla_{\phi} \cdot \nabla f=\Delta f-\nabla \phi \cdot \nabla f .
$$


$\triangleright$ By definition of $D^{s} u$ and using integration by parts, we have

$$
\begin{aligned}
\left(-\Delta_{S} u, u\right) & =2 \int_{\mathbb{R}^{d}}\left|D^{s} u\right|^{2} e^{-\phi} \mathrm{d} x=\frac{1}{2} \sum_{i, j=1}^{d} \int_{\mathbb{R}^{d}}\left(\partial_{i} u_{j}+\partial_{j} u_{i}\right)^{2} e^{-\phi} \mathrm{d} x \\
& =-\frac{1}{2} \sum_{i, j=1}^{d} \int_{\mathbb{R}^{d}} u_{j} \partial_{i}\left(\left(\partial_{i} u_{j}+\partial_{j} u_{i}\right) e^{-\phi}\right) \mathrm{d} x-\frac{1}{2} \sum_{i, j=1}^{d} \int_{\mathbb{R}^{d}} u_{i} \partial_{j}\left(\left(\partial_{i} u_{j}+\partial_{j} u_{i}\right) e^{-\phi}\right) \mathrm{d} x \\
& =-\sum_{i, j=1}^{d} \int_{\mathbb{R}^{d}} u_{j} \partial_{i}\left(\left(\partial_{i} u_{j}+\partial_{j} u_{i}\right) e^{-\phi}\right) \mathrm{d} x \\
& =-\sum_{i=1}^{d} \int_{\mathbb{R}^{d}}\left(u_{i} \Delta u_{i}+u_{i} \partial_{i j} u_{j}\right) e^{-\phi} \mathrm{d} x+\sum_{i=1}^{d} \int_{\mathbb{R}^{d}} u_{i}\left((\nabla \phi \cdot \nabla) u_{i}+2\left(D^{s} u \nabla \phi\right)_{i}\right) e^{-\phi} \mathrm{d} x \\
& =-\int_{\mathbb{R}^{d}} u \cdot\left(\Delta u+\nabla(\nabla \cdot u)-(\nabla \phi \cdot \nabla) u-2 D^{s} u \nabla \phi\right) \mathrm{d} x .
\end{aligned}
$$

so that $-\Delta_{S}$ is given, for an arbitrary vector field $u \in \mathcal{C}_{c}^{\infty}\left(\mathbb{R}^{d} ; \mathbb{R}^{d}\right)$, by

$$
-\Delta_{S} u=-D_{\phi}^{s} \cdot D^{s} u=-\left(\Delta u+\nabla(\nabla \cdot u)-(\nabla \phi \cdot \nabla) u-2 D^{s} u \nabla \phi\right) .
$$

B.3. Gaussian measure. In the normalized centred Gaussian case $\phi(x)=\frac{1}{2}|x|^{2}+\frac{d}{2} \ln (2 \pi)$ corresponding to (5), the basic constants are $C_{\mathrm{P}}=1$ (which is the optimal constant in the Gaussian Poincaré inequality), either $C_{\phi}=1+4 d$ and $C_{\phi}^{\prime}=4 \sqrt{d(1+4 d)}$ if $d \geq 2$, or $C_{\phi}=8$ and $C_{\phi}^{\prime}=8 \sqrt{2}$ if $d=1$, as a limit case.

Let $u(x)=\left(1-x_{2}^{2}, x_{1} x_{2}, 0, \ldots 0\right)^{\perp}$. By elementary computations, we find that

$$
D u=\left(\begin{array}{ccc}
0 & -2 x_{2} & \mathbf{0} \\
x_{2} & x_{1} & \\
& \mathbf{0} & \mathbf{0}
\end{array}\right), \quad D^{s} u=\left(\begin{array}{ccc}
0 & -\frac{1}{2} x_{2} & \mathbf{0} \\
-\frac{1}{2} x_{2} & x_{1} & \mathbf{0}
\end{array}\right), \quad D^{a} u=\left(\begin{array}{ccc}
0 & -\frac{3}{2} x_{2} & \mathbf{0} \\
\frac{3}{2} x_{2} & 0 & \mathbf{0} \\
\mathbf{0} & \mathbf{0}
\end{array}\right)
$$

where $\mathbf{0}$ denotes $2 \times(d-2),(d-2) \times 2$, and $(d-2) \times(d-2)$ null matrices. After integration against the normalized centred Gaussian measure, we have

$$
\langle u\rangle=0, \quad\|u\|^{2}=3, \quad \mathbb{P}(u)=0, \quad \mathfrak{P}(D u)=0=\left\langle D^{a} u\right\rangle, \quad\left\|D^{s} u\right\|^{2}=\frac{3}{2}, \quad\left\|D^{a} u\right\|^{2}=\frac{9}{2}, \quad\|D u\|^{2}=6 .
$$

This proves that $C_{\mathrm{K}}=4$ in $\|D u-\mathfrak{P}(D u)\|^{2} \leq C_{\mathrm{K}}\left\|D^{s} u\right\|^{2}$ and $C_{\mathrm{PK}}=2$ in $\|u-\langle u\rangle-\mathbb{P}(u)\|^{2} \leq C_{\mathrm{PK}}\left\|D^{s} u\right\|^{2}$ are both optimal.

B.4. Estimates for the $D(\Lambda)$-Toolbox and consequences. Here we give some details on the computation of $C_{\mathrm{B}}$ in the proof of Proposition 8 in Section 3.1. Let $A=\|\lfloor\nabla \phi\rceil \nabla f\|, B=\left\|\lfloor\nabla \phi\rceil^{2} f\right\|, Z=\|\xi\|$ and let $c^{\prime 2}=C_{\phi}^{\prime} \geq C_{\phi}=c^{2}$. Inequalities (36) and (37) amount to

$$
B \leq \frac{4}{3} c A+\frac{5}{3} c^{\prime 2} Z \text { and } A^{2} \leq B Z+\frac{1}{2 c} A B+\frac{c^{\prime 2}}{2 c} A Z .
$$

Taking the equality case in the first inequality, we find that

$$
A^{2}-4 A\left(c+c^{\prime 2} / c\right) Z-5 c^{\prime 2} Z^{2} \leq 0
$$

which means that

$$
A \leq\left(2 \sigma+\sqrt{4 \sigma^{2}+5}\right) c^{\prime} Z \quad \text { with } \quad \sigma=\frac{c}{c^{\prime}}+\frac{c^{\prime}}{c} \geq 2 .
$$

On $[2,+\infty)$, the function $\sigma \mapsto \sqrt{4 \sigma^{2}+5} / \sigma$ is monotone non-increasing, so that $\sqrt{4 \sigma^{2}+5} \leq \frac{1}{2} \sqrt{21} \sigma$. Using the monotonicity of $c \mapsto c \sigma$ and $c \leq c^{\prime}$, we also have $c \sigma \leq 2 c^{\prime}$. As a consequence, we have

$$
\begin{aligned}
& A \leq\left(2+\frac{1}{2} \sqrt{21}\right) c \sigma \frac{c^{\prime}}{c} Z \leq(4+\sqrt{21}) \frac{c^{\prime 2}}{c} Z \leq 9 \frac{c^{\prime 2}}{c} Z, \\
& B \leq \frac{1}{3}\left(4 c\left(2 \sigma+\sqrt{4 \sigma^{2}+5}\right)+5 c^{\prime}\right) c^{\prime} Z \leq(7+4 \sqrt{7 / 3}) \leq 14 c^{\prime 2} Z,
\end{aligned}
$$

that is, the bounds (38). Moreover, from $\left\|D^{2} f\right\|^{2} \leq \frac{C}{d}+\frac{1}{2}\left(d+\sqrt{d^{2}+4 C}\right)\|\xi\|^{2}$ with $C=\frac{277}{8}\left(C_{\phi}-1\right) C_{\phi}^{\prime 2}$, we deduce that

$$
C_{\mathrm{B}}=81 \frac{C_{\phi}^{\prime 2}}{C_{\phi}}+196 C_{\phi}^{\prime 2}+\frac{C}{d}+\frac{1}{2}\left(d+\sqrt{d^{2}+4 C}\right) .
$$

B.5. Estimates on various constants. The constants $C_{\phi}$ and $C_{\phi}^{\prime}$ appear in (6) as a consequence of (H2) while the Poincaré constant $C_{\mathrm{P}}$ follows from Assumption (H3). According to (41), the constant in the Poincaré-Lions inequality $(22)$ is given with $C_{\mathrm{B}}$ as in (52) by $C_{\mathrm{PL}}=\left(1+C_{\mathrm{P}}\right)^{2}\left(1+C_{\phi}^{\prime} \sqrt{C_{\mathrm{B}}} / 4\right)^{2}$. From Proposition 5, we know that the strong Poincaré inequality $(21)$ holds for some $C_{\mathrm{SP}} \leq C_{\phi}\left(1+C_{\mathrm{P}}\right)$. As for the other constants in Theorems 1 and 2, we learn from the proofs in Sections 4.1 and 4.2 that

$$
\begin{aligned}
& C_{\mathrm{K}} \leq 1+4 C_{\mathrm{PL}}, \quad C_{\mathrm{PK}} \leq C_{\mathrm{P}} C_{\mathrm{K}}, \quad C_{\mathrm{SPK}} \leq C_{\mathrm{SP}}\left(C_{\mathrm{K}}+3 C_{\phi} C_{\mathrm{PK}}\right), \\
& C_{\mathrm{PK}}^{\prime} \leq C_{\mathrm{PK}}+2 C_{\mathrm{RV}} C_{\mathrm{SPK}} \quad \text { and } \quad C_{\mathrm{K}}^{\prime} \leq C_{\mathrm{K}}\left(1+2 C_{\mathrm{RD}} C_{\mathrm{SP}}\right) .
\end{aligned}
$$


In Section 6.1, Lemma 10, using $C_{\phi}^{\prime \prime}:=C_{\varepsilon}$ as in (H2) with $\varepsilon=1 /\left(2 \sqrt{C_{\mathrm{B}}}\right)$, the constants in (46) are

$$
C_{\mathrm{LPL}} \leq 4\left(1+C_{\mathrm{P}}\right)^{2}\left(1+C_{\phi}^{\prime \prime}\right)^{2} \quad \text { and } \quad C_{\mathrm{RPL}} \leq\left(1+\frac{1}{4} C_{\phi}^{\prime} \sqrt{C_{\mathrm{B}} / C_{\phi}}\right)^{2} .
$$

Finally, the constants in Theorem 4 are given by

$$
C_{\mathrm{K} 0}=1+4 C_{\mathrm{LPL}}, \quad C_{\mathrm{PK} 0} \leq C_{\mathrm{PL}}\left(1+4 C_{\mathrm{LPL}}\right) \quad \text { and } \quad C_{\mathrm{PK} 0}^{\prime} \leq C_{\mathrm{PK} 0}\left(1+2 C_{\mathrm{RV} 0} C_{\phi}\right) .
$$

\section{ACKNOWLEDGMENTS}

This work has been partially supported by the Projects EFI (K.C., J.D., ANR-17-CE40-0030) and Kibord (K.C., J.D., S.M., ANR-13-BS01-0004) of the French National Research Agency (ANR). C.M. and S.M. acknowledge partial funding by the ERC grants MATKIT 2011-2016 and MAFRAN 2017-2022. Moreover C.M. is very grateful for the hospitality at Université Paris-Dauphine.

(C) 2020 by the authors. This paper may be reproduced, in its entirety, for non-commercial purposes.

\section{REFERENCES}

[1] Agmon, S. Lectures on exponential decay of solutions of second-order elliptic equations: bounds on eigenfunctions of $N$-body Schrödinger operators, vol. 29 of Mathematical Notes. Princeton University Press, Princeton, NJ; University of Tokyo Press, Tokyo, 1982.

[2] Ahmed, Z. M., And Stroock, D. W. A Hodge theory for some non-compact manifolds. J. Differential Geom. 54, 1 (2000), $177-225$.

[3] Bakry, D., And Émery, M. Diffusions hypercontractives. In Séminaire de probabilités, XIX, 1983/84, vol. 1123 of Lecture Notes in Math. Springer, Berlin, 1985, pp. 177-206.

[4] Bardos, C., Golse, F., Nguyen, T. T., And Sentis, R. The Maxwell-Boltzmann approximation for ion kinetic modeling. Phys. D 376/377 (2018), 94-107.

[5] Bauer, S., And Pauly, D. On Korn's first inequality for mixed tangential and normal boundary conditions on bounded Lipschitz domains in $\mathbb{R}^{N}$. Ann. Univ. Ferrara Sez. VII Sci. Mat. 62, 2 (2016), 173-188.

[6] BAuer, S., AND Pauly, D. On Korn's first inequality for tangential or normal boundary conditions with explicit constants. Math. Methods Appl. Sci. 39, 18 (2016), 5695-5704.

[7] Carrapatoso, K., Dolbeault, J., Hérau, F., Mischler, S., Mouhot, C., and Schmeiser, C. Special modes and hypocoercivity for linear kinetic equations with full local conservations laws and a confining potential. In preparation.

[8] Ciarlet, P. G. Mathematical elasticity. Vol. I, vol. 20 of Studies in Mathematics and its Applications. North-Holland Publishing Co., Amsterdam, 1988.

[9] Ciarlet, P. G. Linear and nonlinear functional analysis with applications. Society for Industrial and Applied Mathematics, Philadelphia, PA, 2013.

[10] Courtade, T. A., And Fathi, M. Stability of the Bakry-Émery theorem on $\mathbb{R}^{n}$. Journal of Functional Analysis 279,2 (aug 2020), 108523.

[11] Cycon, H. L., Froese, R. G., Kirsch, W., And Simon, B. Schrödinger operators with application to quantum mechanics and global geometry. Texts and Monographs in Physics. Springer-Verlag, Berlin, 1987.

[12] Desvillettes, L., And Villani, C. On a variant of Korn's inequality arising in statistical mechanics. ESAIM Control Optim. Calc. Var. 8 (2002), 603-619 (electronic). A tribute to J. L. Lions.

[13] Desvillettes, L., And Villani, C. On the trend to global equilibrium for spatially inhomogeneous kinetic systems: the Boltzmann equation. Invent. Math. 159, 2 (2005), 245-316.

[14] Dolbeault, J., And Volzone, B. Improved poincaré inequalities. Nonlinear Analysis: Theory, Methods \& Applications 75,16 (nov 2012), 5985-6001.

[15] Duan, R. Hypocoercivity of linear degenerately dissipative kinetic equations. Nonlinearity 24, 8 (Jun 2011 ), $2165-2189$.

[16] Duvaut, G., And Lions, J.-L. Inequalities in mechanics and physics. Springer-Verlag, Berlin-New York, 1976. Translated from the French by C. W. John, Grundlehren der Mathematischen Wissenschaften, 219.

[17] Escobedo, M., and Kavian, O. Variational problems related to self-similar solutions of the heat equation. Nonlinear Anal. 11, 10 (1987), 1103-1133.

[18] Figalli, A. A geometric lower bound on Grad's number. ESAIM Control Optim. Calc. Var. 15, 3 (2009), 569-575.

[19] Friedrichs, K. O. On the boundary-value problems of the theory of elasticity and Korn's inequality. Ann. of Math. (2) 48 (1947), 441-471.

[20] Grad, H. On Boltzmann's H-theorem. J. Soc. Indust. Appl. Math. 13 (1965), 259-277.

[21] Helffer, B., AND Nier, F. Hypoelliptic estimates and spectral theory for Fokker-Planck operators and Witten Laplacians, vol. 1862 of Lecture Notes in Mathematics. Springer-Verlag, Berlin, 2005.

[22] Helffer, B., and Sjöstrand, J. On the correlation for Kac-like models in the convex case. J. Statist. Phys. 74, 1-2 (1994), 349-409.

[23] HÉrau, F., And Nier, F. Isotropic hypoellipticity and trend to equilibrium for the Fokker-Planck equation with a highdegree potential. Arch. Ration. Mech. Anal. 171, 2 (2004), 151-218.

[24] Horgan, C. O. Korn's inequalities and their applications in continuum mechanics. SIAM Rev. 37, 4 (1995), $491-511$.

[25] Johnsen, J. On the spectral properties of Witten-Laplacians, their range projections and Brascamp-Lieb's inequality. Integral Equations Operator Theory 36, 3 (2000), 288-324.

[26] Kato, T. Schrödinger operators with singular potentials. Israel J. Math. 13 (1972), 135-148 (1973).

[27] Kondratiev, V. A., And Oleinik, O. A. On Korn's inequalities. C. R. Acad. Sci. Paris Sér. I Math. 308, 16 (1989), $483-487$. 
[28] Korn, A. Die Eigenschwingungen eines elastischen Körpers mit ruhender Oberfläche. Akad. der Wissensch., Munich, Math. phys. KI. 36 (1906), 351.

[29] Korn, A. Solution générale du problème d'équilibre dans la théorie de l'élasticité, dans le cas ou les efforts sont donnés à la surface. Annales de la Faculté des sciences de Toulouse : Mathématiques 2ième Série, 10 (1908), $165-269$.

[30] KorN, A. Über einige Ungleichungen, welche in der Theorie der elastischen und elektrischen Schwingungen eine Rolle spielen. Krak. Anz. (1909), 705-724.

[31] Ledoux, M. The concentration of measure phenomenon, vol. 89 of Mathematical Surveys and Monographs. American Mathematical Society, Providence, RI, 2001.

[32] LewiCKA, M., AND Müller, S. On the optimal constants in Korn's and geometric rigidity estimates, in bounded and unbounded domains, under Neumann boundary conditions. Indiana Univ. Math. J. 65, 2 (2016), $377-397$.

[33] Mounot, C., Russ, E., And Sire, Y. Fractional Poincaré inequalities for general measures. J. Math. Pures Appl. (9) 95, 1 (2011), 72-84.

[34] Neff, P., Pauly, D., And Witsch, K.-J. Poincaré meets Korn via Maxwell: extending Korn's first inequality to incompatible tensor fields. J. Differential Equations 258, 4 (2015), 1267-1302.

[35] Nitsche, J. A. On Korn's second inequality. RAIRO Anal. Numér. 15, 3 (1981), $237-248$.

[36] Persson, A. Bounds for the discrete part of the spectrum of a semi-bounded Schrödinger operator. Math. Scand. 8 (1960), 143-153.

[37] Reed, M., And Simon, B. Methods of modern mathematical physics. II. Fourier analysis, self-adjointness. Academic Press [Harcourt Brace Jovanovich, Publishers], New York-London, 1975.

[38] Simader, C. G. Essential self-adjointness of Schrödinger operators bounded from below. Math. Z. 159, 1 (1978), 47-50.

[39] Sjöstrand, J. Correlation asymptotics and Witten Laplacians. Algebra i Analiz 8, 1 (1996), $160-191$.

[40] Witten, E. Supersymmetry and Morse theory. J. Differential Geometry 17, 4 (1982), 661-692 (1983).

(K. Carrapatoso) CMLS (CNRS UMR N 7640), École Polytechnique, Institut Polytechnique de Paris, 91128 Palaiseau Cedex, France

Email address: kleber.carrapatoso@polytechnique.edu

(J. Dolbeault) CEREmade (CNRS UMR N 7534), PSL university, Université Paris-Dauphine, Place de Lattre de Tassigny, 75775 Paris 16, France

Email address: dolbeaul@ceremade.dauphine.fr

(F. Hérau) LMJL (CNRS UMR N 6629), 2, Rue de la Houssinière, Université de Nantes BP 92208, F-44322 Nantes Cedex 3, France

Email address: frederic.herau@univ-nantes.fr

(S. Mischler) CEREMAde (CNRS UMR N 7534), PSL university, Université Paris-Dauphine, Place de Lattre de TASSIGNy, 75775 PARIS 16, France

Email address: mischler@ceremade.dauphine.fr

(C. Mouhot) DPMms, Center for Mathematical Sciences, University of Cambridge, Wilberforce Road, CamBRIDGE CB3 0WA, UK

Email address: C.Mouhot@dpmms.cam.ac.uk 